\title{
PAPER
}

\section{Vestibular activation by bone conducted sound}

\section{S Welgampola, S M Rosengren, G M Halmagyi, J G Colebatch}

See end of article for authors' affiliations

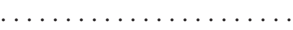

Correspondence to: Dr J G Colebatch, Institute of Neurological Sciences, Prince of Wales Hospital, Randwick, Sydney, NSW 2031, Australia;

i.colebatch@unsw.edu.au

Received 16 September 2002

In revised form

3 January 2003

Accepted

11 January 2003

\begin{abstract}
Objective: To examine the properties and potential clinical uses of myogenic potentials to bone conducted sound.

Methods: Myogenic potentials were recorded from normal volunteers, using bone conducted tone bursts of $7 \mathrm{~ms}$ duration and $250-2000 \mathrm{~Hz}$ frequencies delivered over the mastoid processes by a B 71 clinical bone vibrator. Biphasic positive-negative $(\mathrm{pln} 1)$ responses were recorded from both sternocleidomastoid (SCM) muscles using averaged unrectified EMG. The best location for stimulus delivery, optimum stimulus frequency, stimulus thresholds, and the effect of aging on evoked response amplitudes and thresholds were systematically examined. Subjects with specific lesions were studied. Vestibular evoked myogenic potentials (VEMP) to air conducted $0.1 \mathrm{~ms}$ clicks, $7 \mathrm{~ms} / 250-2000 \mathrm{~Hz}$ tones, and forehead taps were measured for comparison.

Results: Bone conducted sound evoked short latency $\mathrm{p} 1 \mathrm{n} 1$ responses in both SCM muscles. Ipsilateral responses occurred earlier and were usually larger. Mean (SD) pl and $n 1$ latencies were 13.6 (1.8) and 22.3 (1.2) ms ipsilaterally and 14.9 (2.1) and 23.7 (2.7) ms contralaterally. Stimuli of $250 \mathrm{~Hz}$ delivered over the mastoid process, posterosuperior to the external acoustic meatus, yielded the largest amplitude responses. Like VEMP in response to air conducted clicks and tones, $\mathrm{p} \ln 1$ responses were absent ipsilaterally in subjects with selective vestibular neurectomy and preserved in those with severe sensorineural hearing loss. However, plnl responses were preserved in conductive hearing loss, whereas VEMP to air conducted sound were abolished or attenuated. Bone conducted response thresholds were 97.5 (3.9) dB SPL/30.5 dB HL, significantly lower than thresholds to air conducted clicks (131.7 (4.9) dB SPL/86.7 dB HL) and tones (1 14.0 (5.3) dB SPL/106 dB HL).

Conclusions: Bone conducted sound evokes pln 1 responses (bone conducted VEMP) which are a useful measure of vestibular function, especially in the presence of conductive hearing loss. For a given perceptual intensity, bone conducted sound activates the vestibular apparatus more effectively than air conducted sound.
\end{abstract}

T he assessment of patients with vertigo and disequilibrium can be challenging, and the availability of investigations that examine each component of the vestibular apparatus would facilitate a more complete evaluation. Vestibulo-ocular reflexes (VOR) to caloric stimulation and sinusoidal rotation have long been in clinical use as measures of lateral semicircular canal function. More recently, impulsive tests which measure VOR to rapid head accelerations in the plane of each semicircular canal have allowed measurement of individual canal function. ${ }^{1}$ Clinically useful techniques that assess otolith function, however, are few and not widely available. ${ }^{2}$ The click evoked myogenic potential-a relatively recently developed simple, non-invasive test of otolith function-is based on the acoustic sensitivity of the saccule. ${ }^{3}$

Intense clicks delivered through headphones evoke vestibular dependent, biphasic potentials (p13n23) recordable from the sternocleidomastoid (SCM) muscles ipsilateral to the stimulated ear, during tonic activation (vestibular evoked myogenic potentials (VEMP): Colebatch et al, 1994³). Their short onset latency of about $8 \mathrm{~ms}$ suggests that these responses are mediated by an oligosynaptic arc, which probably consists of the primary vestibular afferents synapsing at the vestibular nuclei, the second order vestibulocollic neurones, and the neck motor neurones innervating the SCM. Intense short tone bursts delivered through headphones also evoke VEMP which have similar properties to those evoked by clicks when stimuli of appropriate frequency and duration are used. ${ }^{45}$ Both click evoked and tone burst evoked responses require the integrity of middle ear conductive mechanism to convey the stimulus to the vestibular end organs. ${ }^{36}$ To enable measurement of VEMP in subjects with conductive hearing loss, Halmagyi et al proposed the use of forehead taps delivered through a modified reflex hammer, which probably results in a wave of vibration that is directly conveyed to both labyrinths, circumventing the middle ear conductive apparatus. ${ }^{7}$ This stimulus evokes an initial positivity (pl) followed by two negativities ("n1,n2," see fig l), of which only the first is vestibular dependent. ${ }^{7}$ The precise vestibular afferents stimulated by a forehead tap have not been established, although an otolith contribution is likely, given the similarity of the waveform to that of the click evoked response. Sheykholeslami et al recently reported that tone bursts delivered by a clinical bone vibrator applied over the mastoid process give rise to short latency potentials in the SCM, which were likely to be of vestibular origin. ${ }^{8}$ This new technique holds promise as an alternative to the tap evoked response, as a method of eliciting vestibular dependent responses in subjects with conductive hearing loss. It has the advantage of being a calibrated and reproducible stimulus and therefore should elicit consistent responses and enable the measurement of stimulus thresholds.

The emergence of several related methods of evoking vestibulocollic reflexes necessitates assessment of their relative merits and definition of the place of each these techniques in vestibular function testing. Our aim in the present study was to examine further the properties of responses evoked by bone conducted sound. We confirmed the vestibular dependence and defined the optimum stimulus parameters, methods of

Abbreviations: $A C$, air conducted, $B C$, bone conducted; $E M G$, electromyography; HL, hearing level; NHL, normal hearing level; SCM, sternocleidomastoid muscle; SPL, sound pressure level; VEMP, vestibular evoked myogenic potentials; VOR, vestibulo-ocular reflexes 


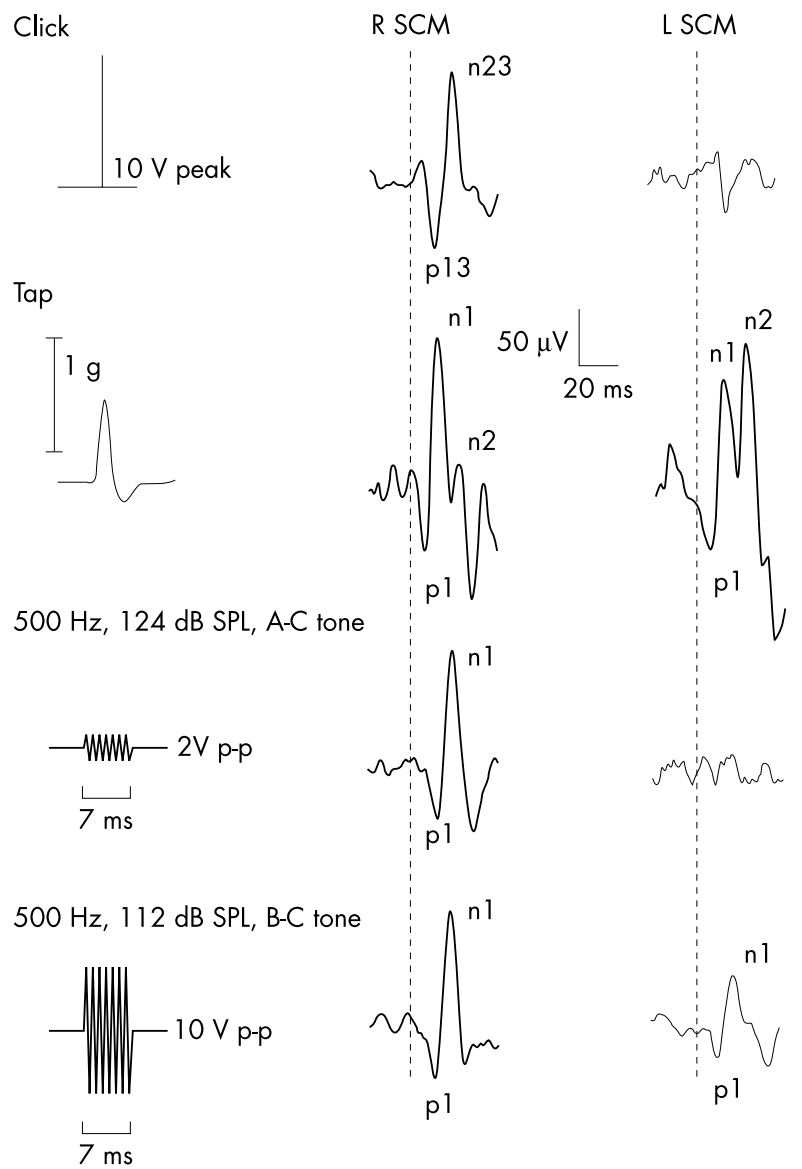

Figure 1 Myogenic potentials to four stimuli in a 25 year old subject. The traces consist of averaged unrectified electromyographic recordings from the left and right sternocleidomastoid muscles (SCM). The thick and thin traces represent responses ipsilateral to and contralateral to the side of stimulation, respectively. $100 \mathrm{~dB} \mathrm{NHL}$ clicks delivered in the right ear evoked p13n23 responses in the ipsilateral SCM. Forehead taps produced bilateral symmetrical vestibular evoked myogenic potentials (VEMP) by activating both labyrinths; these consisted of an initial positivity followed by two negativities $(\mathrm{n} 1, \mathrm{n} 2)$, of which the first is vestibular dependent. Air conducted (AC) tones (124 dB SPL/500 Hz/7 ms), like clicks, give rise only to ipsilateral $\mathrm{pln} 1$ responses. Bone conducted $(B C)$ tones (1 $12 \mathrm{~dB} \mathrm{SPL} / 500 \mathrm{~Hz} / 7 \mathrm{~ms}$ ) result in $\mathrm{p} 1 \mathrm{n} 1$ responses bilaterally; the ipsilateral response being larger and occurring earlier in most subjects. Corrected reflex amplitudes for clicks, taps (averaged for left and right sides), air conducted tones, and bone conducted tones were $1.5,2.1,1.64$, and 2.05 , respectively. Tone amplitudes are plotted to scale; head acceleration is shown for taps.

delivery, and reproducibility of bone conducted responses, and compared them with VEMP evoked by air conducted tones, clicks, and taps in the same subjects.

\section{METHODS}

Ten healthy adults aged 24 to 52 years (five men, five women) were studied after obtaining informed consent and local ethics committee approval.

Myogenic potentials to $7 \mathrm{~ms} / 500 \mathrm{~Hz} / 112 \mathrm{~dB}$ SPL (sound pressure level) tone bursts delivered by a clinical bone vibrator (B71, Radioear Corporation, New Eagle, Pennsylvania, USA) placed on the mastoid process $(3 \mathrm{~cm}$ posterior to the external acoustic meatus) were recorded from all subjects. VEMP to clicks of $0.1 \mathrm{~ms} / 145 \mathrm{~dB}$ SPL $(=100 \mathrm{~dB}$ normal hearing level (NHL)) and $7 \mathrm{~ms} / 500 \mathrm{~Hz}$ tone bursts of $123.5 \mathrm{~dB}$ SPL delivered through headphones (our "standard parameters" for these stimuli) and forehead taps were also measured for compari- son. Although values for air and bone conducted sound are not directly comparable, we have expressed all intensities in $\mathrm{dB}$ SPL.

Subjects were studied lying on a couch with an adjustable backrest, at $45^{\circ}$ from the horizontal plane, while raising their heads against gravity to activate both SCM muscles. Electromyographic (EMG) activity was recorded from surface electrodes over symmetrical sites on the SCM muscles. Active recording electrodes were placed on the junction of the upper and middle thirds of the muscle belly, and reference electrodes on the medial portion of the clavicles. Both rectified and unrectified EMG signals were amplified and bandpass filtered (8 Hz to $1.6 \mathrm{kHz}$ ); EMG was sampled $(5 \mathrm{kHz})$ from $20 \mathrm{~ms}$ before to $100 \mathrm{~ms}$ after the stimulus onset (unless otherwise specified) using a 1401 Plus analogue to digital converter and Sigavg software (Cambridge Electronic Design, Cambridge, UK) on a PC. Evoked responses were measured using unrectified averaged EMG; responses to 256 stimuli were averaged for each ear or side tested. The response amplitudes were measured peak to peak and divided by the mean rectified EMG activity level for the $20 \mathrm{~ms}$ preceding the stimulus onset to correct for background activation ("corrected amplitudes"). For VEMP, the relation between the reflex amplitude and tonic EMG activity is linear (Colebatch et al, 1994 ), and this correction would be expected to control for differences in activation. In the present study, results are presented as both raw (uncorrected) amplitudes and corrected reflex amplitudes. Side to side differences in corrected amplitudes were expressed as an asymmetry ratio (AR); where $A_{1}$ and $A_{s}$ were the larger and the smaller amplitudes of the left and right sides,

$$
A R=100 *\left(\left(A_{1}-A_{s}\right) /\left(A_{1}+A_{s}\right)\right)^{9}
$$

\section{Myogenic potentials evoked by bone conducted sound}

Tone bursts were generated using a Jupiter 2010 function generator (Black Star, Cambridge, UK); these were amplified and gated electronically. The onset and offset of the gate were fixed and were independent of the phase of the input. Bone conducted stimuli were delivered using a B7I clinical bone vibrator previously calibrated using a Bruel and Kjaer (model BMK 4930) artificial mastoid. An initial study was undertaken using stimuli of $7 \mathrm{~ms}$ duration/500 Hz/1 $12 \mathrm{~dB}$ SPL ("standard parameters") delivered at three per second; these were based upon the optimum parameters for air conducted tone bursts. ${ }^{5}$

The effects of varying different parameters (location, tonic activation, frequency) were systematically investigated. Using the standard parameters, myogenic responses were recorded in five subjects with the bone vibrator placed on Fpz, the anterior temporal region (approximately $2 \mathrm{~cm}$ above and $4 \mathrm{~cm}$ anterior to the external acoustic meatus), the mastoid processes ( $3 \mathrm{~cm}$ posterior to the external acoustic meatus), and the external occipital protuberance. The best location for delivery of the stimulus was mapped in greater detail in 10 subjects, using a grid consisting of 12 points around the external acoustic meatus.

To determine whether bone conducted tone burst evoked response amplitudes also increase linearly with EMG activity, responses to standard stimuli were measured in five subjects, while maintaining different degrees of background activation (approximately 20, 40, 60, 80, and $100 \mu \mathrm{V}$ ). Peak to peak amplitudes were plotted against mean rectified EMG activity. In addition, one subject was studied at 15 different levels of activation corresponding to mean rectified EMG levels of 15 $\mu \mathrm{V}$ to $150 \mu \mathrm{V}$. Myogenic potentials were measured in two separate experiments done seven to 10 days apart in 10 subjects to assess between-experiment reproducibility. Reproducibility of tap evoked responses was also examined for comparison.

To determine the optimum stimulus frequency, responses to 250, 500, 1000, and $2000 \mathrm{~Hz}$ bone conducted tone bursts were 
measured in 10 subjects. Stimuli of nearly constant intensities were used (that is, 107.5, 107, 107.5, and 105.5 dB SPL, corresponding to $20,5,10$, and 10 volts peak to peak respectively at 250, 500, 1000, and $2000 \mathrm{~Hz}$ ). These stimuli, generated digitally, were phase locked at $0^{\circ}$ and had $1 \mathrm{~ms}$ rise and fall times and a $7 \mathrm{~ms}$ hold time. In each subject, the optimum stimulus frequency was determined and stimulus thresholds were measured at this frequency using $6 \mathrm{~dB}$ decrements in intensity.

We have previously reported clear effects of age on VEMP amplitudes and thresholds. ${ }^{9}$ Therefore myogenic potentials to bone conducted sound were measured in 10 older subjects aged 63 to 83 years (mean (SD), $71.6(6.7)$ ), using $7 \mathrm{~ms} / 500$ and $1000 \mathrm{~Hz}$ tones of $10 \mathrm{~V} \mathrm{p}-\mathrm{p}$ (equivalent to 112 and $107.5 \mathrm{~dB}$ SPL). These frequencies, at which the bone conductor had its greatest output, were chosen to enable more effective stimulation. Those who had no response to stimuli with these parameters were studied using more intense stimuli of $20 \mathrm{~V} \mathrm{p}-\mathrm{p}$ ( 115 and $112 \mathrm{~dB}$ at 500 and $1000 \mathrm{~Hz}$ ). Stimulus thresholds were also measured. VEMP to air conducted tones with similar parameters were recorded and thresholds measured for comparison.

We measured responses (net potentials) to combined air and bone conducted tones delivered to the same ear at varying degrees of phase advance of the bone conducted tone, using $20^{\circ}$ increments. Air and bone conducted tones $(7 \mathrm{~ms} / 500 \mathrm{~Hz})$ with intensities that evoked responses of comparable amplitudes were used. The amplitude of the net potential to the combined stimuli was measured. Decreases in the net potential were interpreted as evidence of phase cancellation of the stimuli.

\section{Patients with specific lesions}

Responses to clicks (145 dB SPL) and to air and bone conducted tones with the standard parameters were measured in four subjects who had undergone selective unilateral vestibular neurectomy, in six subjects with conductive hearing loss ("air-bone gaps" of 10-40 dB), and in four subjects with profound sensorineural hearing loss. Four subjects (five ears) with the "Tullio" phenomenon secondary to superior semicircular canal dehiscence had stimulus thresholds measured to clicks, air conducted tones, and bone conducted tones. One subject with a bilateral near total vestibulopathy, who had marked reduction of caloric responses and decreased VOR in the planes of all six semicircular canals (Cremer P, personal communication), was studied using air conducted clicks and tones and bone conducted tones.

\section{VEMP evoked by air conducted clicks, tones, and forehead taps}

VEMP to clicks and tone bursts delivered through headphones were measured in all 10 subjects. The clicks of $0.1 \mathrm{~ms}$ duration and $145 \mathrm{~dB}$ SPL ( $=100 \mathrm{~dB}$ NHL) were generated using an ST 10 evoked potential stimulator (Medelec, Surrey, UK) and tone bursts of $2 \mathrm{~V} \mathrm{p}-\mathrm{p}$ intensity $(=123,123.5,124$, and $125 \mathrm{~dB}$ SPL at 250, 500, 1000, and $2000 \mathrm{~Hz}$ ) were generated using the function generator. During each recording, stimuli were presented pseudorandomly in each ear at five per second through calibrated headphones (TDH 49, Telephonics Corp, New York, USA) and responses to 512 stimuli (256 in each ear) were averaged. Frequency tuning properties of air conducted tones were examined using the same methods as for bone conducted tones. Click and air conducted tone thresholds were measured using $5 \mathrm{~dB}$ and $6 \mathrm{~dB}$ decrements, respectively.

Forehead taps were delivered manually through a small gauze pad placed approximately over Fpz, using a nylon handled reflex hammer fitted with an inertial trigger switch. Responses to 64 stimuli were averaged. In subjects who did not have two separate negative peaks $(\mathrm{n} 1, \mathrm{n} 2)$, a slur on the upstroke leading to the negativity or a point at which a clear change in the slope of the upstroke occurred was marked as nl. Data of subjects who had "non-separating peaks" without these features were treated as missing values.

\section{Statistical methods}

SPSS version 9 (SPSS Inc, Chicago, Illinois, USA) was used for all data analyses. To examine the effects of varying location and frequency, repeated measures analysis of variance (ANOVA) with contrasts were done using the corrected reflex amplitudes as the dependent variables and stimulus location or stimulus frequency as fixed factors. A significance level of 0.05 was used, with Bonferroni corrections for multiple comparisons. All results are given as mean (SD) unless otherwise noted.

\section{RESULTS}

Properties of bone conducted responses and comparison with VEMP to other stimuli

All 10 subjects had short latency biphasic positive-negative $(p \ln 1)$ responses to bone conducted tone bursts of standard parameters, which were present bilaterally. Later responses were also present but will not be discussed further. Eight subjects had larger amplitude bone conducted responses recorded from the SCM ipsilateral to the stimulus and smaller ones from the contralateral SCM (see fig 1) and two had the reverse. All subjects had VEMP to clicks and "air conducted" tone bursts of standard parameters recorded from the SCM ipsilateral to the side of stimulation. Seven of the 10 subjects had measurable VEMP to forehead taps recorded from both SCM, two had measurable VEMP on one side (SCM) only. There were four "non-separating" responses (two unilateral and one bilateral).

Bone conducted tones evoked myogenic responses, which had significantly higher average corrected amplitudes (mean (SD), $1.36(0.45))$ recorded from the SCM ipsilateral to the stimulus compared with the contralateral SCM $(0.93(0.66))$ $(\mathrm{p}<0.001)$. Raw amplitudes were 77.0 (26.2) $\mu \mathrm{V}$ ipsilaterally and 55.2 (34.8) $\mu \mathrm{V}$ contralaterally. Peak latencies recorded from the SCM ipsilateral to the stimulus occurred earlier (average pl and nl of 13.6 (1.8) ms and 22.3 (1.2) ms) compared with those recorded over the contralateral SCM (pl and $\mathrm{nl}$ of 14.9 (2.1) and $23.7(2.7) \mathrm{ms}$ ). The range for pl latencies was 11.9 to $16.5 \mathrm{~ms}$ ipsilaterally and 12.5 to $19.6 \mathrm{~ms}$ contralaterally, and that for nl latencies was 20.0 to $26.6 \mathrm{~ms}$ ipsilaterally and 20.1 to $30.2 \mathrm{~ms}$ contralaterally.

The average corrected amplitudes for click, tap, and air conducted tone burst evoked responses were 1.98 (0.48), 2.4 (0.52), and $1.3(0.52) \mu \mathrm{V}$ (raw amplitudes of 130.4 (32.9), 247.0 (61.2), and $78.3(32.8) \mu \mathrm{V}$, respectively). The average peak latencies (p13n23 or plnl) for clicks, taps, and air conducted tones were similar to those reported in previous studies (12.4 (1.2) ms and 20.6 (1.3) ms for clicks; 9.7 (1.2) ms and 18.2 (1.6) ms for taps; and 14.3 (1.3) and 22.9 (1.7) $\mathrm{ms}$ for $500 \mathrm{~Hz}$ air conducted tones)..$^{59}$

Three of the four subjects who had undergone selective vestibular neurectomy as a treatment for intractable vertigo had absent short latency bone conducted responses in the ipsilateral SCM on stimulation of the mastoid on the side of the lesion. On stimulation of the intact side, plnl responses were present on that same side only. One of these three subjects, when stimulated over the operated side, had no response ipsilaterally but a well formed plnl response was recorded from the contralateral SCM, presumably due to bone conduction of the tone to the opposite labyrinth (fig 2). A fourth subject who had undergone selective vestibular neurectomy had a small plnl response recorded from the ipsilateral SCM following stimulation of the side of the lesion, but this finding was not reproducible on repeated recordings. The subject was also found to have residual posterior semicircular canal function on impulsive testing, ${ }^{1}$ suggesting residual nerve function related to the inferior division of the vestibular nerve (Cremer 


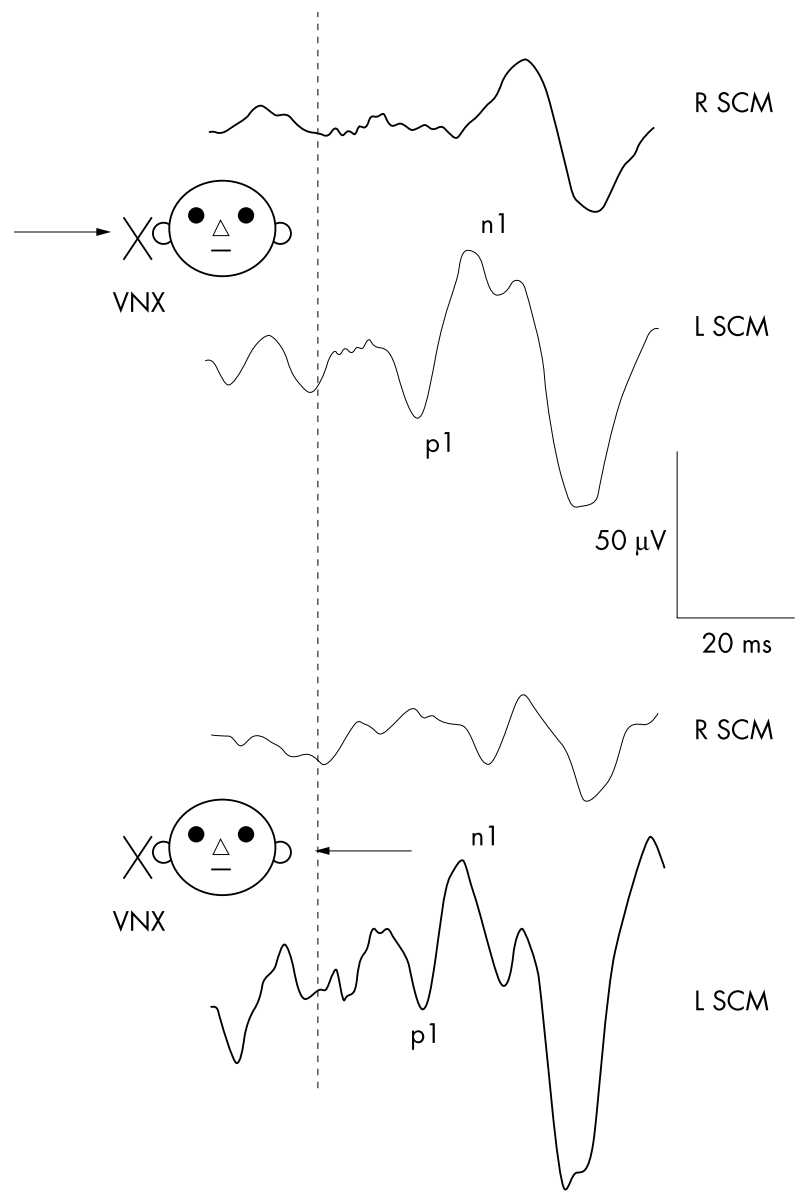

Figure 2 Myogenic potentials to bone conducted tones after right vestibular neurectomy. Upon stimulating over the right mastoid, no pln 1 response is seen over the right sternocleidomastoid (SCM), although a well formed response is present over the left SCM probably secondary to conduction of the tone across the skull (see text). When the unaffected (left) side is stimulated, a response is observed ipsilaterally but no response is observed on the right. Note that later responses were not abolished on the right.

P, personal communication). As with click and tone burst evoked VEMP, short latency responses to bone conducted sound were preserved in the four subjects with severe sensorineural hearing loss. The above findings confirm the conclusions of Sheykholeslami et al, ${ }^{8}$ that short latency plnl myogenic responses to bone conducted sound are vestibular dependent and we shall henceforth refer to them as "bone conducted VEMP".

All five subjects tested for the effect of stimulus location had bone conducted VEMP to the standard stimulus parameters when the bone conductor was placed on the mastoid process ( $3 \mathrm{~cm}$ posterior to the external acoustic meatus). When stimulated over the temporal and occipital regions, ipsilateral bone conducted VEMP were obtainable only in three and two of the five subjects, respectively. Stimulation over Fpz resulted in bilateral responses in three of the five subjects, a unilateral response in one, and absent responses in the other. The average corrected VEMP amplitudes for the mastoid, frontal, anterior temporal, and occipital regions were $1.22,0.32,0.36$, and 0.31 , respectively. Raw amplitudes were $76.0,44.0,14.0$, and $6.5 \mu \mathrm{V}$, respectively. Upon detailed mapping of amplitudes, when the stimulus was applied at 12 different locations in relation to the external acoustic meatus, larger bone conducted VEMP were recorded from locations superior and posterior to the meatus (fig 3). A location $3 \mathrm{~cm}$ behind and 2 $\mathrm{cm}$ above the external acoustic meatus yielded the highest average VEMP amplitude for the 10 subjects, although the
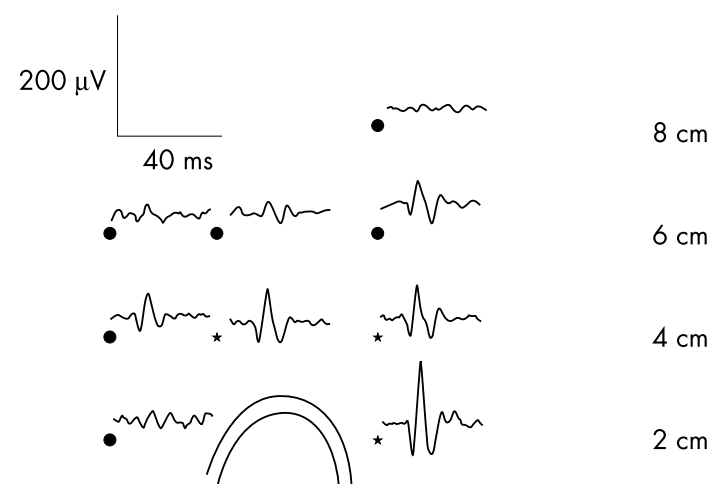

EAM

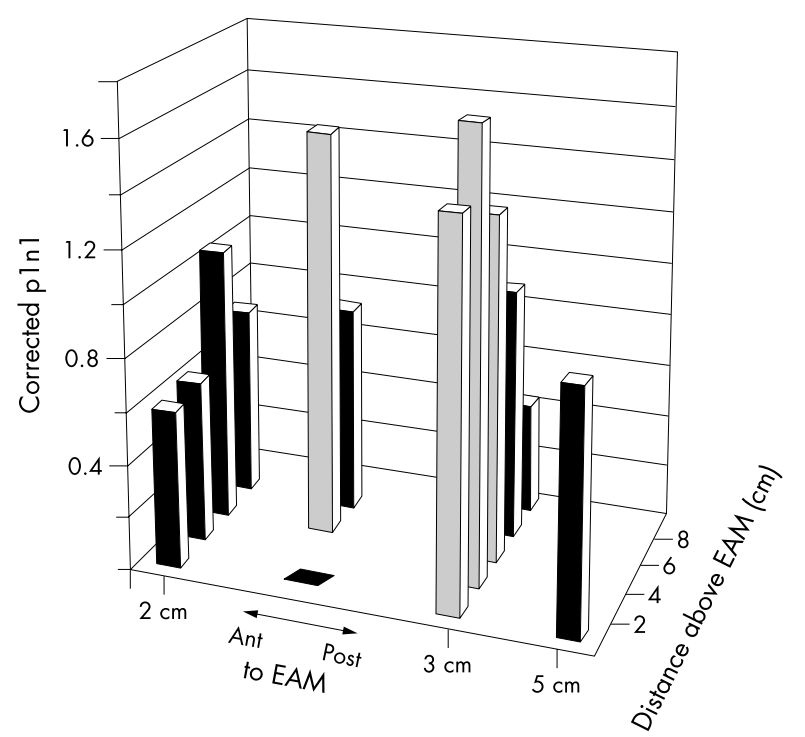

Figure 3 The effect of stimulus location on bone conducted vestibular evoked myogenic potential (VEMP) amplitude. The upper half of the figure shows individual VEMP recorded from one subject's left sternocleidomastoid (SCM) with the bone conductor placed on the left mastoid process and the adjacent temporal bone over 12 locations adjacent to the external acoustic meatus. The bar graph gives average VEMP amplitudes for 10 subjects at each of these locations. The four highest mean amplitudes (pale bars) were observed at the four locations (each marked with an asterisk) closest to the external acoustic meatus posteriorly and superiorly.

best site varied between subjects. A significant effect of location was present on ANOVA $\left(\mathrm{F}_{11,99}=16.5, \mathrm{p}<0.001\right)$. On simple contrasts between VEMP amplitudes at the optimum location and the remaining sites, there were significant differences between it and eight of the remaining 11 locations. There were no significant differences between the amplitude at the optimum site and those at three other locations ("optimum locations"). These formed an inverted L behind and above the external acoustic meatus and were located $3 \mathrm{~cm}$ behind, $3 \mathrm{~cm}$ behind and $2 \mathrm{~cm}$ above, $3 \mathrm{~cm}$ behind and $4 \mathrm{~cm}$ above, and $4 \mathrm{~cm}$ directly above the external acoustic meatus, respectively (fig 3 ).

The peak to peak amplitudes of bone conducted VEMP increased linearly with increasing mean rectified EMG activity 


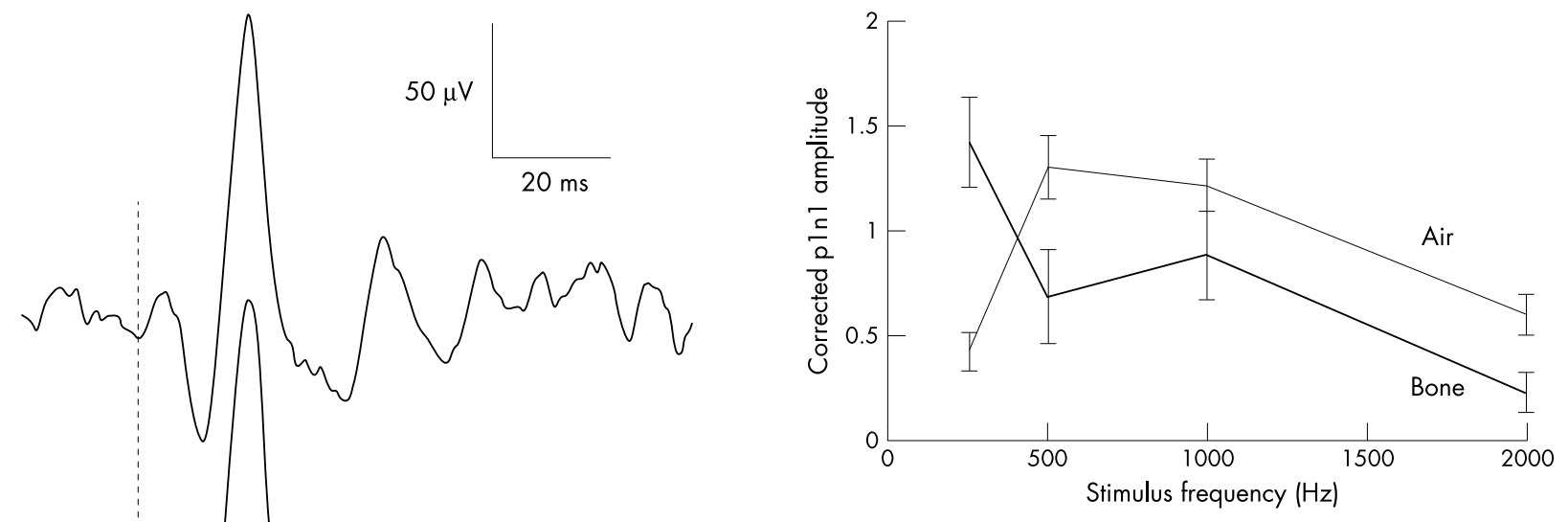

Figure 5 Frequency tuning properties of air and bone conducted tone burst evoked responses. Mean and SEM values of vestibular evoked myogenic potential (VEMP) amplitudes in response to $7 \mathrm{~ms}$ tone bursts of 250,500, 1000, and $2000 \mathrm{~Hz}$ are given; the lines connect average amplitudes at the four frequencies. The thin line represents VEMP to air conducted tone bursts of 123 to $125 \mathrm{~dB}$ SPL and the thick line, VEMP to 105.5 to $107.5 \mathrm{~dB}$ bone conducted tone bursts. Air conducted tones have a tuning curve that peaks between 500 and $1000 \mathrm{~Hz}$ with marked reduction of mean VEMP amplitudes at $250 \mathrm{~Hz}$. Bone conducted VEMP, in contrast, have maximal amplitudes at $250 \mathrm{~Hz}$.

of background activation ( 18 to $155 \mu \mathrm{V}$ ), the raw peak to peak amplitudes varied from 21.1 to $262 \mu \mathrm{V}$ (12-fold). The corrected amplitudes only varied from 1.1 to 1.9 (1.7-fold), confirming that this measure was more constant and less likely to be influenced by EMG activity when compared with raw amplitudes. Correlation of VEMP amplitudes to the standard stimulus obtained on two separate days (approximately one week apart) for 10 subjects resulted in a coefficient of 0.77 $(\mathrm{p}<0.001)$ for bone conducted VEMP $(\mathrm{n}=10)$. A similar correlation coefficient (0.71) was obtained for tap evoked responses $(\mathrm{n}=10)$. The tendency to have larger VEMP recorded from the contralateral SCM, observed in two subjects, was not reproducible between experiments.

\section{Frequency tuning properties and side to side differences}

Average corrected VEMP amplitudes recorded from the ipsilateral SCM for $7 \mathrm{~ms} / 250,500,1000$, and $2000 \mathrm{~Hz}$ bone conducted tones (107 dB nominally) were $1.43(0.2), 0.68$ (0.2), $0.88(0.2)$, and $0.30(0.1)$, respectively (fig 5). Average raw amplitudes were 104.4 (60.2), 58.1 (54.7), 70.2 (47.0), and 24.1 (15.9) $\mu \mathrm{V}$, respectively. Differences between reflex amplitudes measured at the four stimulus frequencies were much greater than the slight differences in stimulus intensity for each frequency (see Methods). In contrast to air conducted tones (fig 5), the largest response was at $250 \mathrm{~Hz}$. On ANOVA with contrasts, average VEMP amplitudes in response to the $250 \mathrm{~Hz}$ stimulus were significantly higher than average amplitudes obtained with the other three stimuli $\left(\mathrm{F}_{3,27}=41.64\right.$, $\mathrm{p}<0.001)$. There was no significant difference between responses to 500 and $1000 \mathrm{~Hz}$ stimuli $(\mathrm{p}=0.56)$. The optimum stimulus frequency was $250 \mathrm{~Hz}$ for 17 sides and 1000 $\mathrm{Hz}$ for three sides. Mean thresholds for bone conducted tones were 97.5 (3.9) dB SPL (30.5 dB HL (hearing level)) when measured using the $250 \mathrm{~Hz}$ stimulus and 100.3 (5.0) dB SPL (42.3 dB HL) using a $500 \mathrm{~Hz}$ stimulus. Thresholds for clicks and air conducted tones were significantly higher at 131.7 (4.9) dB SPL (86.7 dB NHL) and 114 (5.3) dB SPL (106 dB $\mathrm{HL}$ ), respectively (fig 6).

Asymmetry ratios for bone conducted tones were calculated using the VEMP amplitudes recorded from the SCM ipsilateral to the stimulus. The average asymmetry ratios for bone conducted tones were $17.3 \%$ (range 0 to 58.2 ), $38.5 \%$ (0 to 


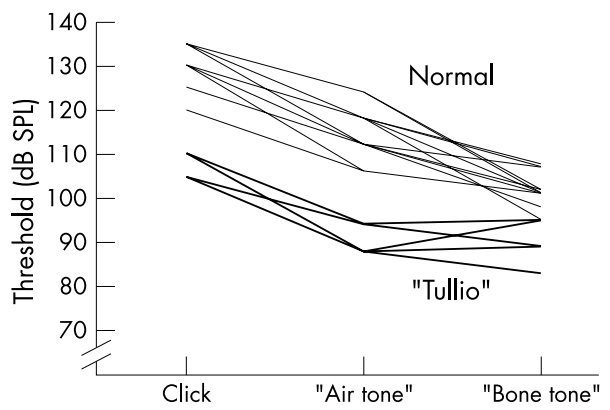

Figure 6 Thresholds to three modes of stimulation. Normal subjects had click thresholds ranging from 120 to $135 \mathrm{~dB}$ SPL, air conducted tone thresholds of 106 to $124 \mathrm{~dB} \mathrm{SPL}$, and bone conducted thresholds of 95 to $107.5 \mathrm{~dB}$ SPL at $500 \mathrm{~Hz}$. The click thresholds for subjects with the Tullio phenomenon were 105 to $110 \mathrm{~dB}$ SPL and air and bone conducted thresholds were 88 to $94 \mathrm{~dB} \mathrm{SPL}$ and 83 to $95 \mathrm{~dB}$ SPL, respectively. While the click and air conducted tone thresholds in Tullio subjects were at least $10 \mathrm{~dB}$ below the lowest normal threshold, those for bone conducted tones approached normal levels in two of the five ears tested.

100 ), $47.8 \%$ (0 to 100 ), and $76.9 \%$ (0 to 100 ) at $250,500,1000$, and $2000 \mathrm{~Hz}$, respectively. Those for air conducted tones were $43.1 \%$ ( 0 to 100 ), $24.9 \%$ ( 0 to 62.7 ), $14.6 \%$ ( 0 to 35.5 ), and $42.8 \%(0$ to 100$)$ at $250,500,1000$, and $2000 \mathrm{~Hz}$. One subject with a short thick neck and SCM muscles that were difficult to palpate had absent bone conducted VEMP to all but the 250 $\mathrm{Hz}$ stimulus (at an intensity of $107 \mathrm{~dB}$ ), and this was responsible for the wide range of asymmetry ratios obtained for the 500 to $2000 \mathrm{~Hz}$ frequencies. The mean asymmetry ratios for VEMP to clicks and taps were $5.4 \%$ (0 to 14.4) and $21.1 \%$ (0 to 42.8).

In the 10 older subjects tested (20 ears), six sides had absent VEMP to $500 \mathrm{~Hz}$ and $1000 \mathrm{~Hz}$ bone conducted stimuli of $10 \mathrm{~V}$ peak to peak (equivalent to 112 and $107.5 \mathrm{~dB}$ SPL, respectively). All these had detectable VEMP when the stimulus was increased to $20 \mathrm{~V}$ peak to peak ( 115 and $112 \mathrm{~dB}$ at 500 $\mathrm{Hz}$ and $1 \mathrm{kHz}$, respectively). VEMP to air conducted tones were absent in seven and five ears at frequencies of 500 and 1000 $\mathrm{Hz}$, respectively. None of these subjects had conductive hearing loss on audiometric testing. Stimulus thresholds at $500 \mathrm{~Hz}$ were 108.6 (4.6) dB SPL (50.6 dB HL) for bone conducted VEMP $(\mathrm{n}=20$ ears $)$ and 121.5 (2.9) dB SPL ( 113.5 $\mathrm{dB} H \mathrm{H})$ for air conducted tones $(\mathrm{n}=15$ ears), both of which were significantly higher than the thresholds obtained in the 10 younger subjects using $500 \mathrm{~Hz}$ stimuli $(\mathrm{p}<0.001$ in both cases).

We were able to demonstrate modest phase cancellation between air and bone conducted tone burst evoked responses. For the three subjects tested, average reflex amplitudes to the air conducted stimulus alone, bone conducted sound alone, and the maximum net potential obtained with the combined stimulus were $1.01,1.06$, and 2.38 , respectively. Thus the maximum net potential approximated the sum of the responses to air and bone conducted sound. For subject 1 , the maximum corrected amplitude for the net potential was 2.77 at intervals of $60^{\circ}$ and $120^{\circ}$; the minimum amplitude was 1.8 at $300^{\circ}$ (35\% attenuation). For subject 2 , the corresponding values were 1.77 at $80^{\circ}$ and 1.26 at $280^{\circ}$ (29\% attenuation), and for subject 3, 2.6 at intervals of $60^{\circ}$ and $160^{\circ}$ and 1.28 at $320^{\circ}$ (51\% attenuation). The amplitudes of the net potentials were normalised to the average net potential of each subject and plotted against the phase interval between the two stimuli (fig 7). A tendency for the net potential to decrease between phase differences of $90^{\circ}$ and $270^{\circ}$ was observed.

\section{Subjects with specific lesions}

Six subjects with conductive hearing loss (eight affected ears) had absent VEMP to $145 \mathrm{~dB}$ SPL clicks and air conducted tone

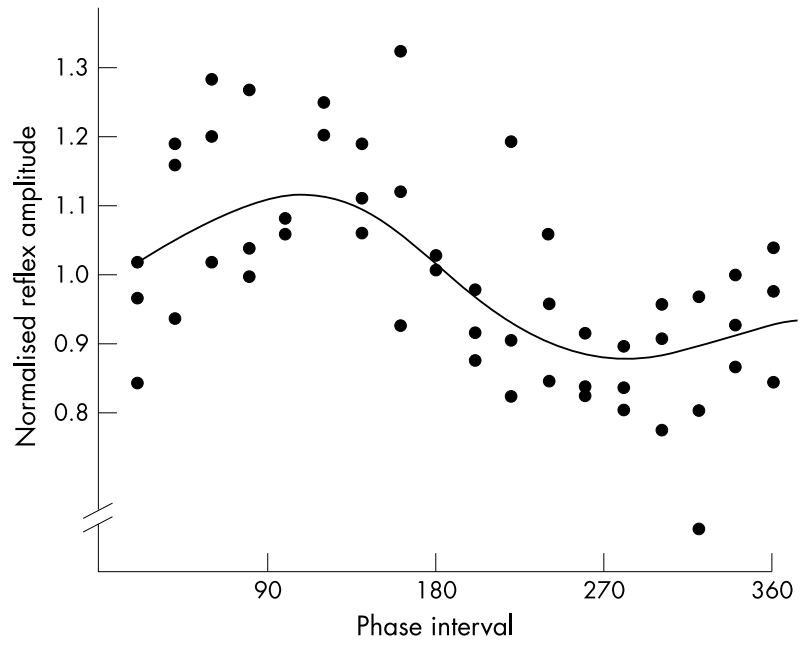

Figure 7 Phase cancellation of air conducted sound evoked responses by bone conducted sound. The scatter plot demonstrates the amplitudes of the "net potentials" of three subjects stimulated by air and bone conducted tones delivered in the right ear at varying phase intervals between $20^{\circ}$ and $360^{\circ}$ (the phase of the tone delivered by the bone conductor always preceded that delivered by the headphones). All amplitudes have been normalised to the individual's average reflex amplitude (average net potential). The line, which represents a LOWESS fit of the data, shows a fall in the amplitude of the net potential between phase differences of $90^{\circ}$ and $270^{\circ}$

bursts of standard parameters on the affected sides. In contrast, VEMP to bone conducted tones of $115 \mathrm{~dB} / 500 \mathrm{~Hz} / 7$ ms were present for in all affected ears.

Four subjects (five ears) with the Tullio phenomenon secondary to dehiscence of the superior semicircular canal ${ }^{10}$ had average thresholds of $107 \mathrm{~dB}$ SPL (62 dB NHL) to clicks, 90.4 dB SPL (82.4 dB HL), to air conducted tones, and $90.2 \mathrm{~dB}$ SPL (32.2 dB HL) to bone conducted tones, all significantly lower than those of normal subjects $(p<0.001)$. The average reduction in thresholds from normal values was 24.7, 23.6, and $10.1 \mathrm{~dB}$ for air conducted clicks and tones and bone conducted tones, respectively (fig 6).

The subject with markedly reduced caloric responses binaurally (maximum SPV on stimulation of either side $=2 \%$ s) as well as marked reduction of VOR to head impulses in the planes of all three semicircular canals on each side, had preservation of VEMP to clicks, air conducted tone bursts, and bone conducted tone bursts of standard parameters delivered to the right side, with corrected amplitudes of $0.97,1.43$, and 0.76 and raw amplitudes of 52.5, 44.9, and 45.1 $\mu \mathrm{V}$, respectively. Upon stimulation of the left ear, VEMP to clicks were absent, and responses to air and bone conducted tones were markedly reduced, with corrected amplitudes of 0.37 and 0.42 and raw amplitudes of 23.8 and $67.0 \mu \mathrm{V}$, respectively.

\section{DISCUSSION}

Although head rotation and acceleration are the adequate physiological stimuli for the vestibular apparatus, nonmechanical stimuli such as loud sound, vibration, and galvanic currents activate primary vestibular afferents and have been used to evoke VEMP. ${ }^{311}$ Vestibular responses to sound have been attributed to stimulation of the saccule, which in other species such as fish and amphibians serves as a primary or secondary organ of hearing. ${ }^{12}{ }^{13}$ Recordings from primary and secondary vestibular neurones in guinea pigs and cats in response to intense clicks or tones have shown increases in neural discharge. ${ }^{14-16}$ Using labelling techniques and responses to physiological stimuli, acoustically responsive afferents been traced to the saccule. ${ }^{15}$ The vestibular apparatus is sensitive to vibration in amphibians ${ }^{17}{ }^{18}$ and mammals. ${ }^{19-22}$ In 
squirrel monkeys, both audio frequency sound and vibration can activate primary afferents from all vestibular end organs, with saccular units showing greater sensitivity than those from the canals to acoustic stimuli but not to vibration. ${ }^{19}$ Vestibular responses such as illusions of self motion and nystagmus following direct vibration of the head using 120-280 Hz stimuli were reported by Lackner and Graybiel, ${ }^{20}$ who attributed these effects to stimulation of the semicircular canals. More recently, low frequency $(100 \mathrm{~Hz})$ vibration of the mastoid process has been found to evoke nystagmus (beating away from the side of the lesion) and shift of the subjective visual horizontal (towards the lesion) in subjects with unilateral vestibular hypofunction, ${ }^{21} 22$ which may reflect asymmetrical vestibular activation.

Bone conducted sound of $7 \mathrm{~ms} / 500 \mathrm{~Hz} / 112 \mathrm{~dB}$ SPL produced short latency plnl potentials in all 10 normal subjects aged 24 to 52 years, responses which were abolished following selective vestibular neurectomy, and preserved despite profound sensorineural hearing loss, and therefore appear to be another form of VEMP. Later responses, sometimes present, were not abolished by vestibular nerve section. Unlike VEMP to clicks and air conducted tones, bone conducted VEMP were often bilateral. Most subjects had larger bone conducted VEMP with earlier peak latencies ( $\mathrm{pl}$ and $\mathrm{nl}$ ranging between 11.9 and $16.5 \mathrm{~ms}$ and between 20.0 and $26.6 \mathrm{~ms}$ ) recorded from the ipsilateral SCM and smaller ones with average peak latencies occurring about $1 \mathrm{~ms}$ later contralaterally ( $\mathrm{pl}$ and $\mathrm{nl}$ ranging between 12.5 and $19.6 \mathrm{~ms}$ and between 20.1 and 30.2 $\mathrm{ms})$. Bone vibration spreads widely throughout the skull, and the contralateral bone conducted VEMP are likely to be responses arising from the opposite labyrinth following stimulus conduction across the skull. Although crossed neural pathways are known to exist, ${ }^{11}$ contralateral bone conducted VEMP clearly differ from the "nlpl" crossed neural responses to galvanic stimulation, ${ }^{11}$ which are of opposite polarity (negative-positive responses) and also have earlier peak latencies compared with the ipsilateral response. The findings in the subject with selective vestibular nerve section indicate that "crossed" bone conducted VEMP do not depend upon activation of the ipsilateral vestibular apparatus. The larger bone conducted VEMP recorded from the contralateral SCM in two subjects further illustrate the vagaries of sound transmission by bone conduction. Similar observations have been made for hearing. Bone conducted sound delivered through the mastoid process has been shown, owing to the asymmetry of skull thickness, to stimulate the contralateral cochlea more effectively in some subjects. ${ }^{23}$ The optimum stimulus frequency for bone conducted VEMP was $250 \mathrm{~Hz}$ for most of the ears tested. Similar optimum frequencies have been reported by Sheykholeslami et al. ${ }^{24}$ Differences between air and bone conducted tone bursts are likely to relate to the different filtering properties of bone and the middle ear as well as to potential differences in the mechanism and specific site of action of the two stimuli. As the B7l vibrator did not permit delivery and accurate calibration of tones of less than $250 \mathrm{~Hz}$ frequency, we were unable to define the tuning curve fully.

While VEMP to air conducted sound are thought to be of saccular origin, the precise origin of the afferents responsible for the bone conducted VEMP is not yet known. Previous experiments which achieved phase cancellation of an air conducted sound by a bone conducted sound ${ }^{25}{ }^{26}$ demonstrated that the two stimuli activate cochlear receptors by similar mechanisms. We have shown that the bone conducted VEMP is vestibular dependent and has similar latency and waveform to the VEMP evoked by air tone bursts. Results obtained from the patient with markedly attenuated canal function imply that bone conducted VEMP arise from the otolith organs. Partial cancellation of responses when air and bone conducted tones were delivered out of phase is consistent with overlap in the receptors stimulated by the two methods. We conclude that the receptors responsible for bone conducted responses arise from the otoliths and in part, at least, from the saccule.
Stimulation over the mastoid yielded bone conducted VEMP more consistently compared with frontal and occipital bones and the temporal bone anterior to the external acoustic meatus. Audiometric studies comparing bone conduction thresholds over the frontal bones and mastoid report lower thresholds over the mastoid. ${ }^{27-30}$ Studies in humans comparing BAER latencies with the stimulus over the frontal, occipital, and temporal bones have reported earlier wave $\mathrm{V}$ latencies to temporal stimulation. ${ }^{31}{ }^{32}$ Our optimum location for stimulus delivery occurred almost always in one of the four locations forming an inverted " $\mathrm{L}$ " shape posterior and superior to the external acoustic meatus; other locations near the meatus gave smaller responses. Sohmer and Freeman reported shorter BAER latencies when the bone vibrator was placed anterior to the external acoustic meatus over the thinnest part of the temporal bone. ${ }^{32}$ We did not find bone conducted VEMP amplitudes to be significantly larger anterior to the external acoustic meatus. This may reflect the more posterior location of the vestibular apparatus compared with the cochlea. Our results indicate that effective stimulation can be delivered at any one of the four optimal locations providing the bone conductor makes good mechanical contact. Symmetrical sites should be used to minimise differences in amplitude between sides.

The perceived loudness of sounds is a function of the middle ear, the cochlea, and the central neural pathways. The standard measure of this property, for continuous sound, is expressed as $\mathrm{AB}$ HL (hearing level) and the relation between $\mathrm{dB}$ HL and $\mathrm{dB}$ SPL (sound pressure level) differs with frequency. The HL reference is not appropriate when the cochlea is not the organ being stimulated by sound, as is the case for VEMP. The reference levels for air and bone conducted sound $(0 \mathrm{~dB})$ are defined differently. For sound in air, $0 \mathrm{~dB}$ SPL is defined as a pressure $(20 \mu \mathrm{P})$, for bone conduction $0 \mathrm{~dB}$ SPL is defined as a force $(1 \mu \mathrm{N})$. The different reference levels prevents direct comparison of air and bone conducted sound. If force is used for comparison, $0 \mathrm{~dB}$ for air is substantially less than $1 \mu \mathrm{N}$. At $1 \mathrm{kHz}, 0 \mathrm{~dB} \mathrm{HL}$ (for sound in air) is close to $0 \mathrm{~dB}$ SPL, implying an extraordinary level of sensitivity, close to the physical limits of detection. Contrasting with sound in air, 0 $\mathrm{dB}$ HL for continuous bone conducted sound is $42.5 \mathrm{~dB}$ higher than the SPL reference for $1 \mathrm{kHz}$ (and therefore approximately 130 times less sensitive). von Békésy ${ }^{33}$ argued that this reduced sensitivity is a desirable characteristic that is in part a consequence of the structure of the cochlea.

The stimulus threshold for air conducted VEMP was $114 \mathrm{~dB}$ SPL $=106 \mathrm{~dB} \mathrm{HL}$, and that for bone conducted VEMP, $97.5 \mathrm{~dB}$ $\mathrm{SPL}=30.5 \mathrm{~dB} \quad \mathrm{HL}$ at $250 \mathrm{~Hz}$ (using standard $\mathrm{HL}$ thresholds). ${ }^{34}$ The difference between hearing thresholds and VEMP thresholds is much larger for air conducted sound than for bone vibration. Bone vibration at a given perceptual intensity is therefore a more effective vestibular stimulus than air conducted sound, implying a relatively greater sensitivity of the vestibular apparatus to bone conducted sound when compared with the cochlea. Subjects with sound induced vestibular symptoms-the Tullio phenomenon-have a low stimulus threshold for VEMP to air conducted clicks, and this has been attributed to an increased sensitivity of the vestibular apparatus to sound at the receptor level, as neural excitability has been shown to be normal in these subjects. ${ }^{35}$ Bone conducted VEMP thresholds, although still significantly lower than those of normal subjects, were not as markedly reduced as the thresholds of VEMP to air conducted clicks in the six subjects with the Tullio phenomenon. A significant proportion of "bone conduction" does in fact travel through the middle ear ${ }^{26}{ }^{36}$ so that some reduction in bone conducted VEMP thresholds would therefore be expected. The vestibular receptors themselves appear to be normal; thus the component of vestibular activation caused by a direct effect on the receptors by distortional vibration and inertial effects on the otoliths may be unaltered, leading to lesser abnormalities than occur with air conducted sound. 
The clinical indications for the use of bone conducted VEMP are similar to those for taps, and they have a similar reproducibility, but they are more consistently present in normal subjects and can be calibrated. Bone conducted VEMP are therefore a suitable alternative to the tap VEMP but they do not replace click evoked VEMP, which are more symmetrical, are highly reproducible $(r=0.9)$ between experiments, ${ }^{9}$ and have a wider separation between normal thresholds and those of subjects with the Tullio phenomenon. All subjects with absent click evoked responses should be tested for VEMP to either bone conducted tones or taps to exclude conductive hearing loss as the cause. When compared with air conducted sound, larger bone conducted stimuli may be safely used, as they do not stimulate the cochlea as effectively. This technique may also be useful in studying older subjects with higher stimulus thresholds. ${ }^{9}$ Absent VEMP to both air and bone conducted sound suggest a lesion along the vestibulocollic reflex pathway. Measurement of VEMP to transmastoid galvanic stimulation ${ }^{11}$ may yield useful information in this context, as this stimulus acts upon the terminal part of the primary vestibular afferents. The preservation of a response to galvanic stimulation when sound evoked responses are absent makes a lesion of the vestibular end organ most likely. ${ }^{37}$

\section{Conclusions}

Bone conducted sound evokes VEMP that provide an additional measure of vestibular function, which is not confounded by conductive hearing loss. They complement information obtained from click VEMP and other techniques currently used in vestibular assessment.

\section{ACKNOWLEDGEMENTS}

This research was supported by a grant from the National Health and Medical Research Council of Australia. MSW received a medical research fellowship from the Garnett Passe and Rodney Williams Memorial Foundation during the period of this study. SMR translated excerpts of the work of Tullio. We thank Dr Toshihisa Murofushi for introducing the technique of measuring bone conducted VEMP, MS C Urban for translating the work of von Békésy, Dr G Colin-Thome for audiological advice, Mr P Bosloper for calibrating the bone conductor, and $\mathrm{Mr}$ Chi Sum Tsang for assistance with patient recruitment. We also acknowledge the expert assistance of Drs Phillip Cremer and Americo Migliaccio in testing three dimensional VOR of two subjects with specific lesions.

\section{Authors' affiliations}

M S Welgampola, S M Rosengren, J G Colebatch, Institute of Neurological Sciences, Prince of Wales Hospital and UNSW Clinical School, Sydney, NSW, Australia

G M Halmagyi, Neurology Department, Royal Prince Alfred Hospital, Camperdown, Sydney Australia

Competing interests: none declared

\section{REFERENCES}

1 Cremer PD, Halmagyi GM, Aw ST, et al. Semicircular canal plane head impulses detect absent function of individual semicircular canals. Brain 1998;121:699-716

2 Halmagyi GM, Curthoys IS. Clinical testing of otolith function. Ann N Y Acad Sci 1999;871:195-204.

3 Colebatch JG, Halmagyi GM, Skuse NF. Myogenic potentials generated by a click-evoked vestibulocollic response. J Neurol Neurosurg Psychiatry 1994;57:190-7

4 Murofushi T, Matsuzaki M, Wu CH. Short tone burst-evoked myogenic potentials on the sternocleidomastoid muscle: are these potentials also of vestibular origin? Arch Otolaryngol Head Neck Surg 1999;125:660-4.
5 Welgampola MS Colebatch JG. Characteristics of tone burst-evoked myogenic potentials in the sternocleidomastoid muscles. Otol Neurotol $2001 ; 22: 796-802$

6 Bath AP, Harris N, McEwan J, et al. Effect of conductive hearing loss on the vestibulo-collic response. Clin Otolaryngol 1999;24:181-3.

7 Halmagyi GM, Yavor RA, Colebatch JG. Tapping the head activates the vestibular system: a new use for the clinical VEMP hammer. Neurology 1995:45:1927-9.

8 Sheykholeslami K, Murofushi T, Kermany MH, et al. Bone-conducted evoked myogenic potentials from the sternocleidomastoid muscle. Acta Otolaryngol 2000;120:731-4.

9 Welgampola MS, Colebatch JG. Vestibulocollic reflexes: normal values and the effect of age. Clin Neurophysiol 2001;112:1971-9.

10 Minor LB, Cremer PD, Carey JP, et al. Symptoms and signs in superio canal dehiscence syndrome. Ann NY Acad Sci. 2001;942:259-73.

11 Watson SR, Colebatch JG. Vestibulocollic reflexes evoked by short-duration galvanic stimulation in man. J Physiol (Lond) 1998:513:587-97.

12 Fay RR, Popper AN. Acoustic stimulation of the ear of the goldfish (Carassius auratus). J Exp Biol 1974;61:243-60.

13 Moffat AJM, Capranica RR. Auditory sensitivity of the saccule in the American toad (Bufo Americanus). J Comp Physiol (A) 1976:105:1-8.

14 McCue MP, Guinan JJ. Acoustically responsive fibers in the vestibular nerve of the cat. J Neurosci 1994; 14:6058-70.

15 Murofushi T, Curthoys IS, Topple AN, et al. Responses of guinea pig neurons to clicks. Exp Brain Res 1995; 103:174-8.

16 Murofushi T, Curthoys IS, Gilchrist DP. Responses of guinea pig vestibular nucleus neurons to clicks. Exp Brain Res 1996;111:49-152.

17 Koyama H, Lewis ER, Leverenz EL, et al. Acute seismic sensitivity in the bullfrog ear. Brain Res 1982;250:168-72.

18 Christensen-Dalsgaard J, Narins PM. Sound and vibration sensitivity of VIllth nerve fibers in the frogs Leptodactylus albilabris and Rana pipiens pipiens. J Comp Physiol (A) 1993;172:653-62.

19 Young ED, Fernandez C, Goldberg JM. Responses of squirrel monkey vestibular neurons to audio-frequency sound and head vibration. Acta Otolaryngol 1977;84:352-60

20 Lackner JR, Graybiel A. Elicitation of vestibular side effects by regional vibration of the head. Aerosp Med 1974;45:1267-72.

21 Hamann KF, Schuster EM. Vibration-induced nystagmus - a sign of unilateral vestibular deficit. ORL J Otorhinolaryngol Relat Spec 1999;61:74-9.

22 Karlberg M, Aw ST, Halmagyi GM, et al. Vibration-induced shift of the subjective visual horizontal: a sign of unilateral vestibular deficit. Arch Otolaryngol Head Neck Surg 2002;1 28:21-7

23 Stephens SDG. Clinical audiometry. In: Beagley HA, ed. Audiology and audiological medicine. Oxford: Oxford University Press, 1981:365-90.

24 Sheykholeslami K, Habiby Kermany M, Kaga K. Frequency sensitivity range of the saccule to bone-conducted stimuli measured by vestibular evoked myogenic potentials. Hear Res 2001;160:58-62.

25 Békésy G von. Experiments in hearing. New York: McGraw Hill, 1960:127-203.

26 Tonndorf J. A new concept of bone conduction. Arch Otolaryngol 1968;87:595-600

27 Whittle LS. A determination of the normal threshold of hearing by bone conduction. J Sound Vib 1965:2:227-48.

28 Barber SP, Rose DE. Bone conduction oscillator placement in testing hearing of selected groups of children. Am J Ment Defic 1969;73:666-72.

29 Weatherton MA, Goetzinger CP. A report on bone conduction thresholds at the mastoid and forehead in normals. Audiology 1971;10:23-9.

30 Haughton PM, Pardoe K. Normal pure tone thresholds for hearing by bone conduction. BrJ Audiol 1981;15:113-21.

31 Yang EY, Rupert AL, Moushegian G. A developmental study of bone conduction auditory brainstem response in infants. Ear Hear 1987:8:244-51.

32 Sohmer $\mathbf{H}$, Freeman S. The latency of auditory nerve brainstem evoked responses to air- and bone-conducted stimuli. Hear Res 2001; 160:111-13

33 von Békésy $\mathbf{G}$. The structure of the middle ear and the hearing of one's own voice by bone conduction. J Acoust Soc Am 1948;21:217-32.

34 Standards Australia. Acoustics-instrumentation for audiometry. Part 1 Reference zero for the calibration of pure-tone bone conduction audiometers. AS/NZS 1591.1. Sydney, 1995: www.standards.com.au

35 Watson SR, Halmagyi GM, Colebatch JG. Vestibular hypersensitivity to sound (Tullio phenomenon): structural and functional assessment Neurology 2000;54:722-8.

36 Tonndorf J. Bone conduction. Studies in experimental animals. Acta Otolaryngol 1966:suppl 213.

37 Murofushi T, Takegoshi H, Ohki M, et al. Galvanic-evoked myogenic responses in patients with an absence of click-evoked vestibulo-collic reflexes. Clin Neurophysiol 2002;113:305-9. 


\section{PostScript}

\section{LETTERS}

\section{Remission of progressive multifocal leucoencephalopathy in SLE after treatment with cidofovir: a 4 year follow up}

Progressive multifocal leucoencephalopathy (PML) is an opportunistic infection caused by human polyomaviruses such as the JC virus. It usually occurs as a severe complication of immunosuppression in patients with primary disorders of the immune system or secondary impairment of immune function, for example, after iatrogenic states of immunosuppression. PML usually takes a rapidly progressive course and advances to death within 1 to 18 months. Today, PML is mainly seen in AIDS, while previously it was typically found in patients with granulomatous, neoplastic, or infectious diseases. In granulomatous diseases particularly, PML is thought to occur as a result of iatrogenic states of immunosuppression, but it is also seen in patients aggressively treated with immunosuppressive agents for systemic lupus erythematosus (SLE). ${ }^{12}$ PML progresses to death in most of these patients even after withdrawing immunosuppressive therapy. ${ }^{2}$ Therefore additional therapy, aimed at supporting a more rapid restoration of immune function his warranted.

\section{Case report}

We report a 40 year old woman diagnosed with SLE at the age of 20 years, based on four American College of Rheumatology criteria (erythema, arthritis, elevated antinuclear antibodies, and anti-dsDNA antibodies). Owing to neuropsychiatric lupus (the patient had experienced several psychotic episodes) with suspected vasculitic changes on cerebral magnetic resonance imaging (MRI), the patient had undergone 12 cycles of cyclophosphamide pulse therapy in 1995/96 followed by immunosuppressive treatment with mycophenolate mofetil in 1998, and azathioprine in 1999. Follow up cerebral MRI scans at that time were normal.

In January 2001, she again developed psychotic episodes and an initially mild ataxia. She had repeatedly been put on low doses of corticoids but on no other immunosuppressive therapy during the previous 2 years. MRI revealed a lesion in the left cerebellum, which was hyperintense on $\mathrm{T}_{2}$ and hypointense on $\mathrm{T}_{1}$ weighted images. No lesions were seen in the cerebral hemispheres. Central nervous system manifestation of SLE was suspected, although the patient revealed only moderate signs of SLE activity (elevated anti-dsDNA antibodies, slightly decreased complement levels C3c and $\mathrm{C} 4$, increased erythrocyte sedimentation rate, but normal $\mathrm{C}$ reactive protein). There were no signs of severe immunosuppression; laboratory data showed normal levels of immunoglobulins and only slightly decreased lymphocytes, especially CD8+ T lymphocytes. The patient received two pulses of cyclophosphamide and high doses of corticosteroids to reduce the presumed cerebral SLE activity. In addition, she received antipsychotic medication.

While the psychosis was readily controlled by this treatment, the patient deteriorated neurologically. She developed a severe, disabling, rapidly progressive, left sided hemiataxia. and was unable to walk. A control MRI of the brain in February 2001 revealed a progression of the lesion in the left cerebellum and a new lesion in the middle cerebellar peduncle. The lesions again presented as hyperintense on $T_{2}$ and hypointense on $T_{1}{ }^{-}$ weighted images (fig 1A). Owing to the neurological deterioration after initiation of immunosuppression and the presentation of the lesions on MRI, PML was considered as a differential diagnosis. PCR revealed JC virus DNA in the cerebrospinal fluid (CSF). As cerebral SLE and PML require an intense but divergent therapy, a brain biopsy was obtained from the cerebellar lesion. Histopathology confirmed the diagnosis of PML. HIV tests were negative, $T$ cell counts were normal, and signs of malignancy were lacking. Immunosuppression was discontinued. Because PML is usually lethal in patients with SLE even after omission of immunosuppression, ${ }^{12}$ we considered options for an active antiviral therapy. There was evidence from several reports in AIDS patients that cidofovir, an inhibitor of viral DNA polymerase, may reduce the size of PML lesions and thus prolong survival. ${ }^{3}$ Lacking therapeutic alternatives we therefore administered intravenous cidofovir $(5 \mathrm{mg} / \mathrm{kg}$ body weight $)$ at initially bi-weekly intervals, after obtaining informed consent. After the third and fourth cycle, the patient improved dramatically. She was able to walk again and only showed a mild residual ataxia. MRI revealed reduction of the lesions in the cerebellum and middle cerebellar peduncle with no new sites of active disease (fig 1B). PCR for JC virus DNA in the CSF was now negative. The treatment with cidofovir was continued with longer intervals (8-12 weeks). The therapy was generally well tolerated. After the fifth cycle, mildly increased creatinine levels were found After one cycle with $4 \mathrm{mg}$ cidofovir/ $\mathrm{kg}$ body weight, kidney function was quickly normalised and the following cycles could be administered at the initial dosage. Fourteen months after initiation of the treatment, the patient had completed the 10th cycle of therapy with no signs of disease activity. MRI scans of the brain showed further regression of the lesion with no signs of

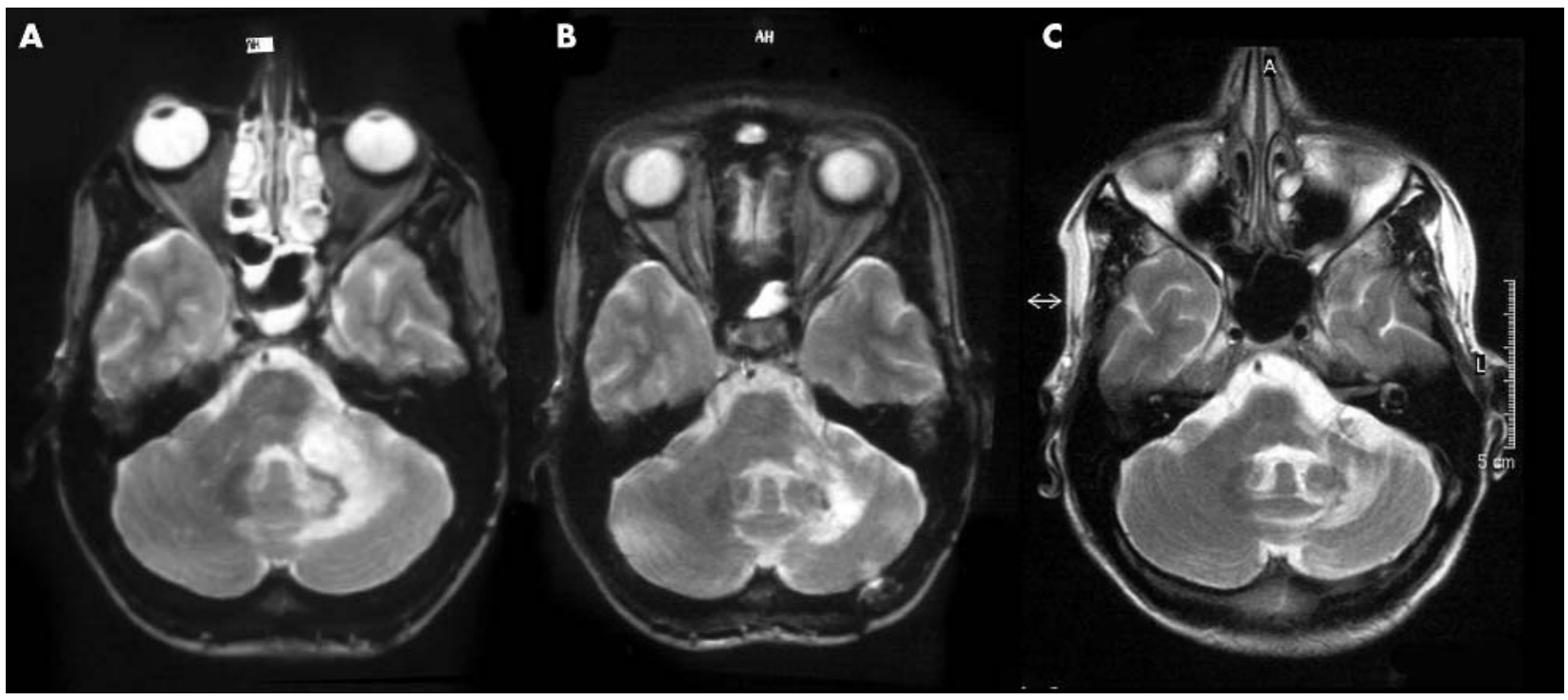

Figure 1 T2 weighted MRIs showed (A) extended white matter lesions in the middle cerebellar peduncle and left cerebellum, February 2001; (B) regression of the lesions after four cycles of cidofovir, July 2001; and (C) further regression with residual gliotic lesions, July 2004. 
active inflammation (not shown), and cidofovir treatment was discontinued. At present, (4 years follow up after the first treatment and 2.5 years after the last cycle of cidofovir), the patient still shows no signs of disease activity. CSF PCR for JC virus DNA remains negative, and a recent MRI scan of the brain was unchanged (fig 1C). As of March 2005 , the patient lives at home, is able to walk, and is independent.

\section{Discussion}

We report a patient with SLE who survived PML after treatment with cidofovir and discontinuation of immunosuppression. First evidence for possible efficacy of cidofovir in the treatment of PML in a patient with SLE was presented in a case report. Discontinuation of immunosuppression and treatment with cidofovir resulted in reversal of JC virus positivity and stabilisation of MRI lesions. However, the patient died due to serious kidney failure.

It remains unclear whether the improvement in both patients was induced or supported by cidofovir or whether it could have been acquired by discontinuation of immunosuppression alone. However, patients with PML in SLE usually die after discontinuation of immunosuppression alone. ${ }^{12}$ Interestingly, our patient did not show signs of severe immunosuppression at the point of manifestation of PML. These observations may suggest a predisposition of patients with SLE to PML that may not be explained by their immunosuppression alone.

We conclude that cidofovir should be offered to SLE patients developing PML due to immunosuppression in addition to withdrawal of immunosuppressive therapy, as death is likely without antiviral therapy. Cidofovir may be effective against PML caused by non-AIDS related states of immunosuppression.

R Reilmann, T Imai, E B Ringelstein Department of Neurology, University of Münster, Münster, Germany

M Gaubitz

Department of Internal Medicine B, University of Münster

T U Niederstadt

Department of Radiology, University of Münster

W Paulus

Department of Neuropathology, University of Münster

I W Husstedt

Department of Neurology, HIV Clinic, University of Münster

Correspondence to: Dr R Reilmann, Department of Neurology, University Clinic Münster (UKM) University of Münster, Albert-Schweitzer Strasse 33, 48129 Münster, Germany r.reilmann@uni-muenster.de

doi: 10.1136/jnnp.2004.057588

Competing interests: none

\section{References}

1 Kinoshita $M$, Iwana K, Shinoura $H$, et al. Progressive multifocal leukoencephalopathy resembling central nervous system systemic lupus erythematosus. Clin Exp Rheumatol 1998;16:313-15.

2 Arbusow V, Strupp M, Samtleben W, et al.

Progressive multifocal leukoencephalopathy as a result of immunosuppressive therapy. Dtsch Med Wochenschr 1999;124:653-6.

3 De Luca A, Giancola ML, Ammassari A, et al. Potent anti-retroviral therapy with or without cidofovir for AIDS-associated progressive multifocal leukoencephalopathy: extended followup of an observational study. $J$ Neurovirol $2001 ; 7: 364-8$.

4 Roberts MT, Carmichael A, Lever AM. Prolonged survival in AIDS-related progressive multifocal leucoencephalopathy following anti-retroviral therapy and cidofovir. Int J Antimicrob Agents 2003;21:347-9.

5 Salmaggi I, Maccagnano E, Castagna A, et al. Reversal of CSF positivity for JC virus genome by cidofovir in a patient with systemic lupus erythematosus and progressive multifocal leucoencephalopathy. Neurol Sci 2001;22:17-20.

\section{Dramatic improvement in non- AIDS related progressive multifocal leucoencephalopathy}

Progressive multifocal leucoencephalopathy (PML) is a rare disorder occurring when a strain of papovavirus (JC virus) infects the central nervous system. It results in a generally quick and fatal outcome. It is associated with cell mediated immune deficient diseases but some few cases were reported in immunocompetent hosts. Since 1981, it has been commonly associated with AIDS. In AIDS related PML, long term survival without real neurological improvement has been reported in patients treated with highly active antiretroviral therapy (HAART). ${ }^{1}$ Few cases of improvement with cidofovir or cytosine arabinoside have been described in AIDS related or non-AIDSrelated $\mathrm{PML}^{2}{ }^{3}$ but in larger trials in AIDS related PML, no clinical benefit was found. ${ }^{4}$ As a whole, the treatment of this progressive demyelinating disease remains controversial, in particular in the rare cases of non-AIDSrelated PML. We describe a patient with an underlying haematological disease, without clear cut immune cell deficiency, who developed rapidly progressive PML. The patient showed clinical, virological, and imaging improvement when treated with an association of intravenous and intrathecal cytosine arabinoside combined with intravenous cidofovir.

A 48 year old man presented with progressive multiple lymphadenopathies, hepatosplenomegaly, weight loss, and blood cell count abnormalities. Fine needle aspiration cytology of lymphadenopathy diagnosed marginal zone B cell lymphoma. There was also bone marrow and blood proliferation. A few weeks after the diagnosis, the patient noticed rotatory vertigo and visual problems suggestive of a right homonymous hemianopia. Because of dissemination and the large tumour mass, chemotherapy (CHOP: cyclophosphamide, doxorubicin, vincristine and prednisone) was started one month after the onset of neurological symptoms. Following the first course of chemotherapy, his neurological symptoms worsened, and language disorders appeared. No real immunodeficiency was shown-the absolute CD4+ count was $549 / \mathrm{mm}^{3}$ (normal $858 \pm 260$ ), and there was discrete hypogammaglobulinaemia (4.6 g/l; normal 5-12). The patient was HIV and HTLVl seronegative. Cerebral magnetic resonance imaging showed a non-contrast-enhancing lesion in the left occipital white matter (fig 1A).

Cerebrospinal fluid (CSF) analysis was normal apart from a moderate increase in
CSF protein $(0.7 \mathrm{~g} / \mathrm{l})$. Suspicion of PML was confirmed by a positive polymerase chain reaction (PCR) for JC virus DNA. Chemotherapy was discontinued (after one cycle) but neurological symptoms worsened rapidly and the patient developed a right hemiplegia, global aphasia, alexia and agraphia, apraxia, and cortical blindness concurrently with MRI deterioration (fig lB).

Three months after his first symptoms, treatment was started with intravenous aracytine $2 \mathrm{mg} / \mathrm{kg} / \mathrm{d}$ for five days every three weeks, combined with intrathecal aracytine (30 mg) weekly and intravenous cidofovir $5 \mathrm{mg} / \mathrm{kg} / \mathrm{d}$ once every two weeks. The main adverse effect of this treatment was grade IV bone marrow toxicity, inducing spacing in the rhythm of treatment administration. One week after treatment onset, the patient stabilised and after one month began improving. After three months, he had recovered completely from his hemiplegia, and had significant improvement in his aphasia and cortical blindness. Right hemianopia and minor alexia without agraphia persisted. This dramatic improvement was confirmed by cerebral imaging, by the absence of JC virus DNA detection in CSF, and by a specific response of CD4+ T cells against JC virus. We decided to continue subcutaneous aracytine $2 \mathrm{mg} / \mathrm{kg} / \mathrm{d}$ for five days monthly and intravenous cidofovir twice weekly. Fifteen months after treatment onset, the patient was ambulatory and cerebral MRI continued to improve (fig 1C). Lymphoma tumour burden did not clearly change during the treatment.

\section{Comment}

We report the favourable outcome of a patient with non-AIDS-related PML treated with a combination of intravenous and intrathecal aracytine and cidofovir. As the patient was not immunocompromised and had not received immunosuppressive treatment at PML onset, risk factors for the occurrence of PML are unclear. Treatment led to a rapid clinical and radiological improvement which was long lasting despite treatment delay and the patient's worrying clinical condition at treatment onset. Dose and administration schedules of cytarabine and cidofovir were derived from reports suggesting individual beneficial effects of these drugs. ${ }^{2} 3$ To our knowledge, these drugs have not been used in combination before. In comparison with previous reports, the present case suggests a more rapid and prolonged effect of this therapeutic combination than with either aracytine or cidofovir treatment alone. This efficacy may be explained by a synergy between the drugs and by their different routes of administration. We thought that the improvement in our patient was related to the treatment because there was a temporal link with treatment onset and because of the radiological features (the rare cases of PML stabilising without specific treatment have usually been associated with an inflammatory response to the virus, indicated by contrast enhancement on imaging).

The main limiting factor of this treatment was bone marrow toxicity. During the periods of immune deficiency, the patient's neurological condition did not deteriorate, suggesting that PML occurrence in this patient was linked to a qualitative defect of CD4 cells rather than to their absolute count. Immunological studies have shown that JCV specific CD4-T cell responses play a 

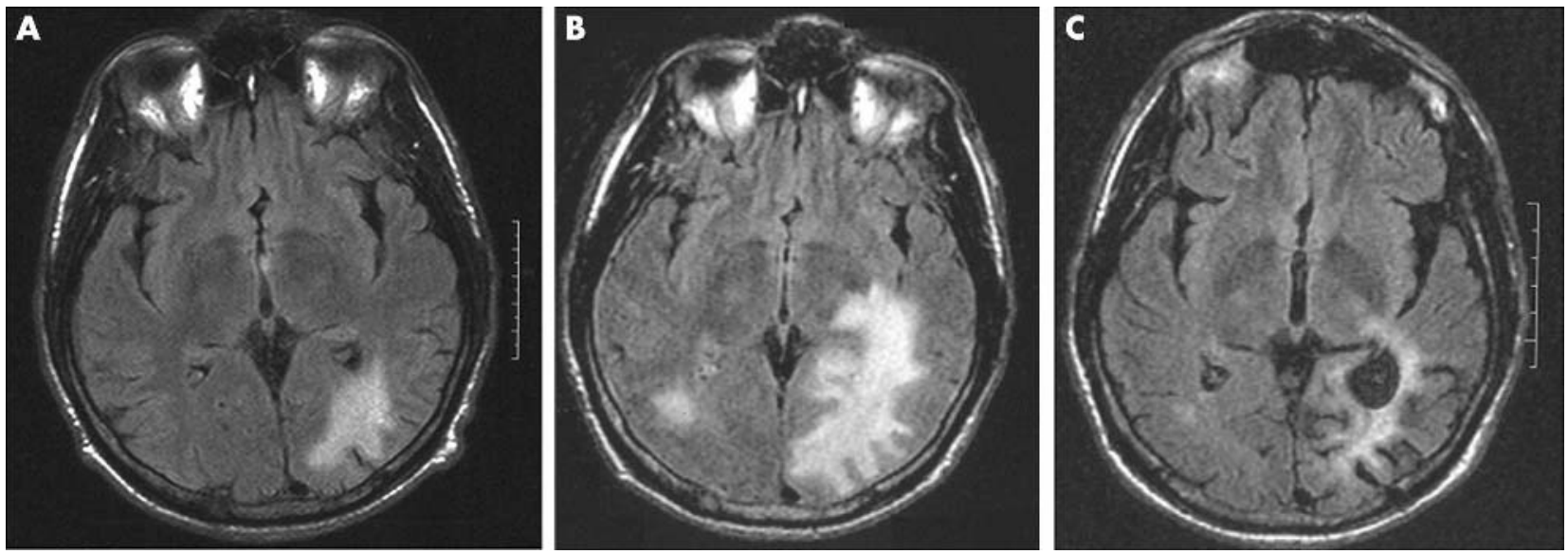

Figure 1 evolution of cerebral magnetic resonance imaging (MRI) before and during treatment. (A) The first cerebral MRI, two months after the first neurological symptoms. Flair weighted axial images (TR $9000 \mathrm{~ms}$; TE $114 \mathrm{~ms}$ ) showing high signal intensity lesion in the left occipital white matter with respect to cortical grey matter. (B) Brain MRI one week after treatment onset. Flair weighted axial images (TR $10002 \mathrm{~ms}$; TE $160 \mathrm{~ms}$ ) showing extension of the lesions. At that time, extension of the lesions was greater, with bilateralisation of the lesions in both occipital white matter and extension through temporal white matter. (C) Brain MRI three months after treatment onset. Flair weighted axial images (TR $10002 \mathrm{~ms}$; TE $160 \mathrm{~ms}$ ) showing dramatic improvement under treatment. Hyperintense signal has diminished in both occipital lobes, but cerebral atrophy had appeared in the left occipital and temporal cortex. TE, time of echo; TR, time of repetition.

major role in the control of PML development; for example, in one study ${ }^{5}$ no specific $\mathrm{T}$ cell response was demonstrated in a series of 14 patients before treatment, whereas nine of 10 survivors recovered specific immunity. Our patient thus probably had no specific $\mathrm{T}$ cell response against JCV before treatment, but recovered a moderate but significant response while on treatment, possibly explaining PML regression. Such a restoration of $\mathrm{T}$ cell response can be achieved by HAART in AIDS. This observation suggests that the combination of aracytine and cidofovir could have had a similar action in restoring a specific $\mathrm{T}$ cell response against JCV in our patient. A direct effect of these nucleosidic analogues against JC virus DNA may also explain the rapid clinical and radiological improvement in our patient.

Despite its substantial bone marrow toxicity, this observation suggests that the new association of intrathecal and intravenous aracytine with intravenous cidofovir could be useful in patients with PML, particularly those with an underlying haematological disease. It is worth noting that bone marrow toxicity did not lead to deterioration of the neurological status of the patient, supporting the view that a specific defect in CD4 function is more important than the absolute CD4 count. The dramatic improvement observed in our patient warrants further prospective studies testing this drug combination.

\section{Acknowledgements}

We thank Dr Yacine Taoufik, Department of Immunology, Kremlin-Bicêtre Hospital, for his help with the immunological data and critical discussion.

K Auré, A Béhin, F Louillet, C Lafitte, M Sanson Service de Neurologie Mazarin, Groupe Hospitalier Pitié-Salpêtrière, Assistance Publique-Hôpitaux de Paris, Paris, France

J P Vernant

Service d'Hématologie, Groupe Hospitalier Pitié-

Salpêtrière

Correspondence to: Dr Anthony Béhin, Federation de Neurologie-Mazarin, Groupe hospitalie
Pitié-Salpêtrière, 47-83 Boulevard de l'hôpital, 75013 Paris Cedex, France; anthony.behin@psl.ap-hopparis.fr

doi: 10.1136/jnnp.2004.061408

Competing interests: none declared

\section{References}

1 Gasnault J, Taoufik Y, Goujard C, et al. Prolonged survival without neurological improvement in patients with AIDS-related progressive multifocal leukoencephalopathy on potent combined antiretroviral therapy. J Neurovirol 1999;5:421-9.

2 Aksamit AJ. Treatment of non-AIDS progressive multifocal leukoencephalopathy with cytosine arabinoside. J Neurovirol 2001;7:386-90.

3 Herrlinger U, Schwarzler F, Beck R, et al. Progressive multifocal leukoencephalopathy: cidofovir therapy in three patients with underlying hematological disease [letter]. J Neurol 2003;250:612-14.

4 Hall CD, Dafni U, Simpson D, et al. Failure of cytarabine in progressive multifocal leukoencephalopathy associated with human immunodeficiency virus infection. N Engl J Med 1998;338:1345-51.

5 Gasnault J, Kahraman M, de Goer de Herve MG, et al. Critical role of JC virus-specific CD4 T-cell responses in preventing progressive multifoca leukoencephalopathy. AIDS 2003;17:1443-9

\section{Diaphragmatic paralysis and respiratory failure as a complication of Lyme disease}

There have been five recorded cases of diaphragmatic paralysis as a complication of neuroborreliosis. ${ }^{1-5}$ Here we report another case of Lyme meningoradiculitis, caused by an identified tick, leading to bilateral diaphragmatic paralysis with an abbreviated course on treatment.

\section{Case report}

A 59 year old female presented with a recent history of abdominal pain and falls because of a weakness in her right leg. She had been complaining of flu-like symptoms with twitches in her back and pain in her right side for a month. She gave a history of recently having been bitten by ticks whilst gardening. There was no history of any recent rash. On the day of presentation, she complained of a mild cough, reduced appetite, abdominal distension, constipation, and dysuria. She was a lifelong smoker but was generally healthy.

At presentation her blood pressure was 206/107 mm Hg. There was some epigastric tenderness. She had bruising on her right leg that she associated with the falls.

The chest radiograph on admission was unremarkable. Abdominal $x$ ray showed dilated loops of small bowel and a loaded colon. Her only blood abnormality was hyponatraemia at $121 \mathrm{mmol} / \mathrm{l}$. She was admitted for further investigations.

On day 3 of admission she became increasingly short of breath and on examination had decreased bibasal air entry. On day 4 her respiratory rate was $25 / \mathrm{min}$ and arterial blood gases (ABG) demonstrated hypoxaemia but adequate ventilation with $\mathrm{pH} 7.51, \mathrm{Po}_{2}$ $6.7 \mathrm{kPa}$, and $\mathrm{PCO}_{2} 4.7 \mathrm{kPa}$. Her chest radiograph showed left basal changes. On day 5 her $\mathrm{PCO}_{2}$ had risen to 6.8; she was admitted to the intensive care unit and non-invasive ventilatory support was commenced. She had a decreased inspiratory pressure and a decreased vital capacity. She was noted to have absent gag reflex and poor swallow and on day 6 was intubated to protect against aspiration pneumonia. The patient remained fully conscious and co-operative, easily triggering the ventilator but requiring significant inspiratory pressure support of $20 \mathrm{~cm} \mathrm{H}_{2} \mathrm{O}$

Neurological examination demonstrated right hip and knee extensor weakness $(2 / 5)$, absent right knee jerk, and a loss of sensation on her left lateral thigh. Because she lived in a known endemic area we thought about Lyme disease, but we also considered differential diagnoses such as Guillain-Barre syndrome, listeriosis, and acute poliomyelitis. We commenced treatment with doxycycline whilst awaiting the results of further investigations. Around this time the patient indicated a small black lesion on her upper abdomen that was removed and on closer examination was identified as tick mouthparts (fig l) 


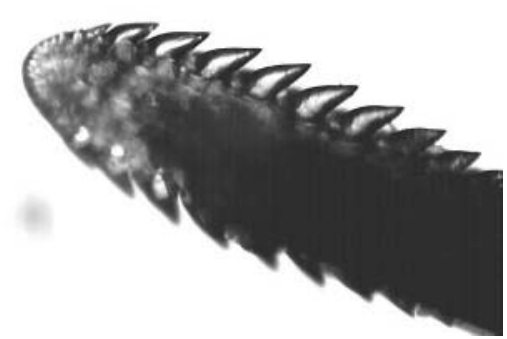

Figure 1 Photomicrograph of tick recovered from patient, showing tick mouthparts.

Given her smoking history and persistent hyponatraemia, a chest computerised tomography was performed which showed only left lower lobe collapse and a small left pleural effusion. Bronchoscopy was unremarkable. Chest ultrasound screening showed only minimal movement of both diaphragms.

Cerebrospinal fluid analysis (CSF) demonstrated a white cell count 181 cells $/ \mathrm{mm}^{3}$ (100\% mononuclear), red cell count 22 cells/ $\mathrm{mm}^{3}$, glucose $2.3 \mathrm{mmol} / \mathrm{l}$ (serum glucose $6.8 \mathrm{mmol} / \mathrm{l}$ ), and protein $0.96 \mathrm{~g} / \mathrm{l}$. Immunological analysis of the CSF was not done because an insufficient sample was obtained. Brain magnetic resonance imaging (MRI) was normal. Spinal MRI and electromyography were not carried out. Immunoglobulin $M$ and immunoglobulin G antibodies to Borrelia burgdorferi were detected in serum, and at this point intravenous ceftriaxone $(2 \mathrm{~g}$ for 30 days) was commenced.

Over the next 7 days her strength increased and a repeat ultrasound of the diaphragm on day 16 of admission showed marked improvement with the right dome moving normally and some residual left sided weakness associated with overlying lung consolidation.

The patient was weaned from the ventilator and extubated following a total of 22 days of respiratory support. She underwent intensive physiotherapy and has made an uneventful recovery. One year later she complained of mild shortness of breath on lying flat with an exercise tolerance of 1 mile on the flat. On examination she had hyperaesthesia in her right leg with slightly brisk knee reflexes (previously absent right knee jerk). She had persistent bilateral diaphragmatic paralysis demonstrated on chest ultrasound screening. Pulmonary function tests showed a $40 \%$ reduction between erect and supine vital capacity measurements.

\section{Discussion}

The first case of diaphragmatic paralysis as a complication of Lyme disease was reported in 1986: a 73 year old male, treated with ampicillin and netilmicin, who required ventilation for 3 months and then died after receiving treatment for a pulmonary embolism. ${ }^{1}$ Another four cases have been reported in patients between the ages of 39 and 68 , all of whom were treated with either doxycycline or ceftriaxone and two of whom required ventilation due to respiratory failure. $^{1-5}$ All patients were well at follow up, although one patient had persistent phrenic paralysis 6 months after treatment.
In all previous cases of diaphragmatic palsy as a complication of Lyme disease, either the patient reported dyspnoea or hypoxia was noted on ABG. The diagnosis of phrenic nerve palsy was made by the following methods: hemidiaphragm elevation, fluoroscopic screening of diaphragmatic movements, or electrical stimulation of phrenic nerves. ${ }^{1-5}$ Our patient had a lymphocytic meningitis with sensory and motor neuropathies including bilateral phrenic nerve palsies. Diaphragmatic paralysis due to Lyme disease was diagnosed on the basis of clinical features, chest ultrasonography, the presence of the tick head, and serology indicating a recent infection with $B$ burgdorferi as well as a rapid response to antibiotic therapy.

The clinical diagnosis of Lyme disease may be supported by serologic testing. B burgdorferi antibody tests may be negative in early infection, but patients are usually seropositive at or shortly after presenting with neurological symptoms. In some patients, antibodies against $B$ burgdorferi may be detectable in CSF slightly earlier than serum. Culture and $B$ burgdorferi deoxyribonucleic acid detection using polymerase chain reaction may also be used but were not in our case.

The three patients reported in the literature with respiratory failure caused by neuroborreliosis were ventilated for 3 months, 1 month, and 13 months, respectively, whilst our patient required ventilation for only 22 days. ${ }^{124}$ We speculate that early recognition of the possibility of Lyme disease and appropriate treatment shortened our patient's acute illness.

In conclusion, it is important to consider Lyme disease in the differential diagnosis of acute respiratory failure - with or without erythema migrans.

\section{Acknowledgements}

We would like to thank Mr P R Randell of the Microbiology Department, St Richard's Hospital, Chichester, UK and Dr Susan O'Connell of the Department of Microbiology and Public Health Laboratory, Southampton General Hospital, Southampton, UK.

R A Abbott, S Hammans, M Margarson, B M Aji St Richard's Hospital, Chichester, UK

Correspondence to: Rachel A Abbott, St Richard's Hospital, Spitalfield Lane, Chichester, West Sussex PO19 6SE, UK; rachel.abbott@nhs.net doi: 10.1136/jnnp.2004.046284 Competing interests: none declared

\section{References}

1 Melet $M$, Gerard A, Voiriot $P$, et al. Méningoradiculonévrite mortelle au cours $d^{\prime}$ une maladie de Lyme. Presse Med 1986;5:2075.

2 Sigler S, Kershaw P, Scheuch R, et al. Rspiratory failure due to Lyme meningoradiculitis. Am J Med 1997:103:544-547.

3 Faul JL, Ruoss S, Doyle RL, et al. Diaphragmatic paralysis due to Lyme disease. Eur Respir J 1999; 13:700-702

4 Winterholler M, Erbguth FJ. Tick bite induced respiratory failure. Intensive Care Med 2001;27:1095.

5 Gomez de la Torre R, Suarez del Villar R, Alvarez Carreno $\mathrm{F}$, et al. Parálisis diafragmática y artromialgia por enfermedad de Lyme. An Med Interna (Madrid) 2003;20:47-9.

\section{Benign paroxysmal positional vertigo (BPPV) predominantly affects the right labyrinth}

We read with great interest the article "Benign paroxysmal positional vertigo predominantly affects the right labyrinth", by M von Brevern et al, ${ }^{1}$ which prompted us to review our data of the last 10 years (19952004).

A total of 661 patients, referred to the ear, nose, and throat department or to the neurology department, were diagnosed as having benign paroxysmal positional vertigo (BPPV) in its various forms. The pathology was located in the posterior canal in 477 patients, in the horizontal canal in 142, and in the anterior canal in 22. Multiple canals were affected in 20 patients (table 1).

The right ear was 1.50 times more frequently involved than the left. The predominance of the right ear was seen in all types of BPPV (table 1).

Hence, our data confirm the preponderance of right sided BPPV. The predilection of right sided BPPV was seen in all variants. Horizontal canal BPPV was observed in $22 \%$, confirming our previous data. ${ }^{2}$ This number is higher than in other series. ${ }^{3}$ The apogeotropic form should not be considered as a rarity as it contributes to $23 \%$ of horizontal canal BPPV in our series and 38\% in the series of Casani et al. ${ }^{3}$

W Damman, R Kuhweide Ear, Nose and Throat Department, AZ St Jan, B-8000 Brugge, Belgium

I Dehaene Department of Neurology, AZ St Jan, B-8000 Brugge, Belgium
Table 1 Laterality of the affected ear in different forms of BPPV

\begin{tabular}{|c|c|c|c|c|c|c|}
\hline \multirow[b]{2}{*}{ No. of patients } & \multirow[b]{2}{*}{$\begin{array}{l}\text { Posterior } \\
\text { canal }\end{array}$} & \multicolumn{2}{|c|}{ Horizontal canal } & \multirow[b]{2}{*}{$\begin{array}{l}\text { Anterior } \\
\text { canal }\end{array}$} & \multirow[b]{2}{*}{$\begin{array}{l}\text { Multiple } \\
\text { canals }\end{array}$} & \multirow[b]{2}{*}{ Totals } \\
\hline & & Geotropic & $\begin{array}{l}\text { Apogeo- } \\
\text { tropic }\end{array}$ & & & \\
\hline $\begin{array}{l}\text { Right } n=379 \\
\text { Left } n=253 \\
\text { Bilateral } n=29\end{array}$ & $\begin{array}{l}266 \\
185 \\
26 \\
72 \%\end{array}$ & $\begin{array}{l}61 \\
48 \\
0 \\
77 \% \\
\text { geotropic }\end{array}$ & $\begin{array}{l}23 \\
10 \\
0 \\
23 \% \\
\text { apogeo- } \\
\text { tropic }\end{array}$ & $\begin{array}{l}15 \\
7 \\
0 \\
3 \%\end{array}$ & $\begin{array}{l}14 \\
3 \\
3 \\
3 \%\end{array}$ & $\begin{array}{l}57 \% \\
38 \% \\
5 \% \\
100 \%\end{array}$ \\
\hline
\end{tabular}

Total $\mathrm{n}=661$ 
Correspondence to: Dr I Dehaene, Department of Neurology, AZ St Jan, Ruddershove 10, B-8000 Brugge, Belgium; ides.dehaene@azbrugge.be

$$
\text { doi: 10.1136/jnnp.2005.065912 }
$$

Competing interests: none declared

\section{References}

1 von Brevern M, Seelig T, Neuhauser H, et al. Benign paroxysmal positional vertigo predominantly affects the right labyrinth. J Neurol Neurosurg Psychiatry 2004;75:1487-8.

2 De la Meilleure G, Dehaene I, Depondt $M$, et al. Benign paroxysmal positional vertigo of the horizontal canal. J Neurol Neurosurg Psychiatry 1996:60:68-71.

3 Casani AP, Vannucci G, Fattori B, et al. The treatment of horizontal canal positional vertigo: our experience in 66 cases. Laryngoscope 2002; 1 12:172-8.

4 Brandt Th. Benign paroxysmal positioning vertigo. In: Brandt T, ed. Vertigo. London: Springer Verlag, 1999:251-83.

\section{Aciclovir induced posterior leucoencephalopathy}

Aciclovir is an extremely effective agent for the treatment of herpes simplex encephalitis and varicella-zoster infections in immunocompromised individuals. ${ }^{1}$ Encephalopathy induced by aciclovir is an infrequent but well recognised adverse effect of aciclovir. ${ }^{2}$ The predisposing factors to aciclovir induced encephalopathy (AIE) include age, acute or chronic renal failure, and other neurotoxic drugs. $^{2}$ Tremors $(40-58 \%)$, disorientation $(40-50 \%)$, agitation $(22-38 \%)$, hallucinations $(25 \%)$, and delirium $(25 \%)$ are common presentations of AIE, whereas seizures $(10 \%)$, cerebellar ataxia $(11 \%)$, sensory symptoms $(9 \%)$, speech disorders $(9 \%)$, fever $(3 \%)$, and cranial nerve palsies $(0 \%)$ are much less frequent. ${ }^{2}$ The supportive diagnostic criteria for AIE include a temporal association between the symptoms and aciclovir use, as well as acellular cerebrospinal fluid (CSF) in cases without herpes simplex or varicella-zoster encephalitis. In the majority of cases symptoms develop within 72 hours of starting aciclovir treatment, although up to 120 days has been reported. ${ }^{2}$ The clinical recovery may take several days (five days in $57 \%$ of cases) following discontinuation of aciclovir. ${ }^{2}$ The EEG typically shows diffuse slow wave activity rather than focal abnormalities. ${ }^{2}$
The radiological features are not well described in AIE. Case reports have identified multifocal white matter signal abnormalities involving the cerebellum, pons, and periventricular region as well as evidence of vascular encephalopathy on MRI. ${ }^{2}{ }^{3}$ We report a case of AIE with MRI features consistent with posterior leucoencephalopathy with clinical and radiological improvement following the discontinuation of aciclovir, along with raised serum and CSF concentrations of aciclovir and 9-carboxymethoxymethylguanine (CMMG), the main metabolite of aciclovir.

\section{Case report}

A 47 year old women with a 15 month history of cANCA $^{+}$glomerulonephritis and chronic end stage renal failure (serum creatinine 957 $\mu \mathrm{mol} / \mathrm{l})$, managed on continuous ambulatory peritoneal dialysis, azathioprine $(100 \mathrm{mg} /$ day), and prednisolone (10 mg/day), developed a mid-thoracic varicella-zoster rash. She was given a reduced dose of intravenous aciclovir (250 mg three times daily). Within 48 hours she became agitated, developed visual hallucinations and drowsiness (Glasgow coma scale (GCS): overall 6; eye 1 , motor 4, verbal 1). On admission to the intensive care unit she was apyrexial, the highest blood pressure recorded was 180/104 while agitated, and fundoscopy was unremarkable. The tendon reflexes were exaggerated, with extensor plantar responses. Oculocephalic and corneal reflexes as well as spontaneous respiration were present.

Laboratory investigations showed a haemoglobin of $9.7 \mathrm{~g} / \mathrm{l}$, white cell count $5.8 \times 10^{9} /$ l, urea $22 \mathrm{mmol} / \mathrm{l}$, ESR $90 \mathrm{~mm} / \mathrm{h}$, C reactive protein $27 \mathrm{mg} / \mathrm{l}$, albumin $22 \mathrm{~g} / \mathrm{l}$, ammonia 10 $\mathrm{mmol} / \mathrm{l}$, and cANCA negative. Computed tomography of the head, done immediately after she became obtunded, showed posterior white matter hypodensities. The lumbar CSF contained no white cells and four red blood cells per $\mathrm{mm}^{3}$, with a protein of $0.63 \mathrm{~g} / \mathrm{l}$ and a $\mathrm{CSF} /$ serum glucose ratio of 3.9/6.4. The opening pressure was $41 \mathrm{~cm}$. CSF cultures, including culture for acid fast bacilli, were negative. CSF testing by polymerase chain reaction was negative for herpes simplex virus I and II, varicella-zoster virus, cryptococcal antigen, and JC virus. An EEG showed excessive slow wave activity. Magnetic resonance imaging (MRI) of the brain and an MR venogram (done after five days on aciclovir) showed symmetrical posterior white matter changes predominantly in the occipital and parietal lobes (fig $\mathrm{IA}$ and $\mathrm{lB}$ ) without evidence of venous sinus thrombosis, gadolinium enhancement, or matched defects in diffusion weighted images.

On admission, azathioprine was stopped and intravenous methylprednisolone $(500 \mathrm{mg} /$ day for three days) was started for presumed CNS vasculitis. There was no improvement 72 hours after this treatment and after eight days of aciclovir. Aciclovir was discontinued when AIE was suspected, and peritoneal dialysis was maintained. Within 48 hours of discontinuation of aciclovir, the GCS improved (eye score 3, motor score 6 , verbal score 3) and neurological examination showed bilateral upper limb postural tremor, which resolved over 24 hours. The Addenbrooke's score was 77/100. Repeat MRI (fig 1C, 1D) showed a significant improvement. Analysis of aciclovir and CMMG levels in the serum and CSF showed high values, at levels generally associated with neurotoxicity. ${ }^{4}$ The serum aciclovir and CMMG concentrations were, respectively, as follows:

- 5 days post-aciclovir initiation: $34.9 \mu \mathrm{mol} /$ $\mathrm{l}$ and $91.7 \mu \mathrm{mol} / \mathrm{l}$;

- 6 days post-aciclovir: $35.4 \mu \mathrm{mol} / \mathrm{l}$ and $148.5 \mu \mathrm{mol} / \mathrm{l}$;

- 7 days post-aciclovir: $17.2 \mu \mathrm{mol} / \mathrm{l}$ and $141 \mu \mathrm{mol} / \mathrm{l}$.

The CSF aciclovir and CMMG concentrations four days post-aciclovir initiation were, respectively, $5.99 \mu \mathrm{mol} / \mathrm{l}$ and $2.25 \mu \mathrm{mol} / \mathrm{A}$ (CMMG levels are not detectable in CSF unless there is neurotoxicity (Helldén A submitted for publication)), supporting a diagnosis of AIE. ${ }^{4}$

\section{Comment}

The clinical presentation of our patient, her rapid recovery, and the CSF and EEG findings are characteristic of AIE in cases without herpes simplex or varicella-zoster encephalitis. ${ }^{25}$ The lack of significantly raised blood pressure or papilloedema excludes hypertensive posterior leucoencephalopathy. $\mathrm{ANCA}^{+}$vasculitis causing posterior leucoencephalopathy has been reported but only in the presence of severe hypertension. AIE, an infrequent but well recognised adverse effect of aciclovir, has until now been diagnosed
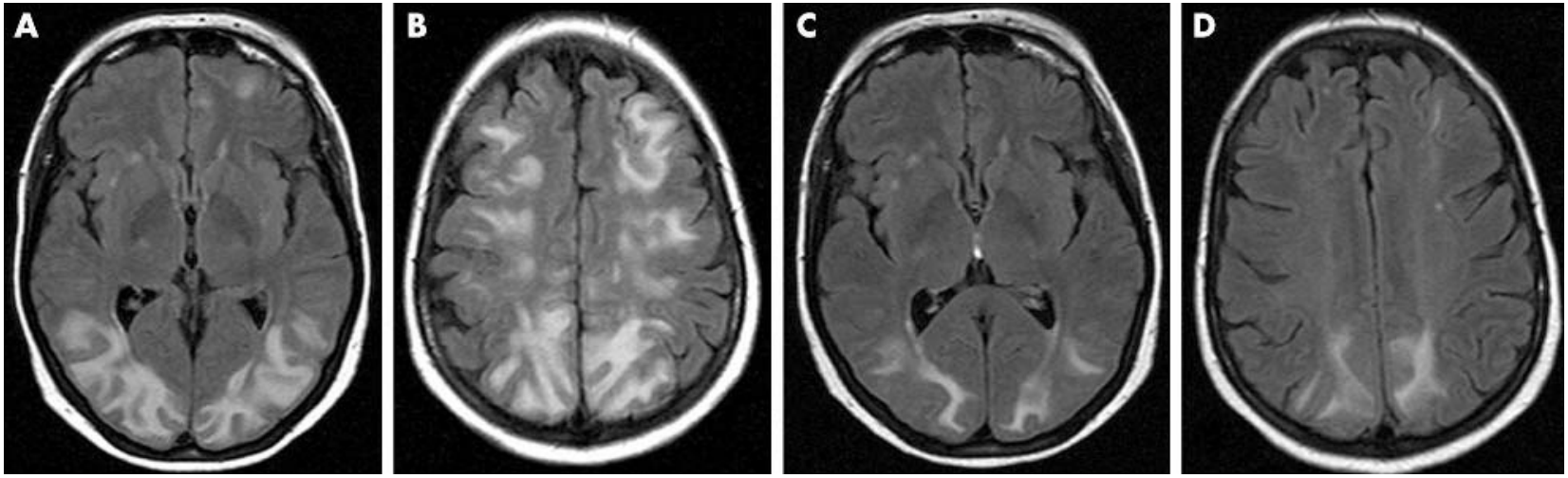

Figure 1 Fluid attenuated inversion recovery (FLAIR) magnetic resonance imaging (MRI) of the brain done five days after initiation of aciclovir treatment, showing features consistent with posterior leucoencephalopathy (panels A and B). Repeat MRI five days after discontinuation of aciclovir showed a marked improvement (panels $C$ and D). 
mainly on clinical features. ${ }^{25}$ Factors predisposing to AIE include age, acute or chronic renal failure, and other neurotoxic drugs. The diagnosis is facilitated by analysis of aciclovir and CMMG in serum and CSF. In cases with renal failure, the half life of aciclovir extends from 3 to 20 hours and as a result aciclovir is metabolised to CMMG by alcohol and aldehyde dehydrogenases. ${ }^{4}$ At present, reliable dose recommendations are not available for patients with renal failure.

In a case study of 93 patients, mainly with renal failure, ${ }^{4}$ we found mean (SD) serum aciclovir concentrations of 21.0 (30.7) $\mu \mathrm{mol} / \mathrm{l}$ (in 49 patients with neurotoxicity) and 7.2 (6.7) $\mu \mathrm{mol} / \mathrm{l}$ (in 44 asymptomatic patients receiving aciclovir), while CMMG concentrations were 34.1 (39.4) $\mu \mathrm{mol} / \mathrm{l}$ in patients with neurotoxicity and 4.7 (4.7) $\mu \mathrm{mol} / \mathrm{l}$ in asymptomatic patients. CMMG levels of $>10 \mu \mathrm{mol} / \mathrm{l}$ seemed to be associated with neurotoxicity. A high CMMG level is a strong predictor of AIE in patients with renal failure. ${ }^{4}$ Haemodialysis is effective in clearing aciclovir and CMMG, and to a lesser extent so is peritoneal dialysis. ${ }^{4}$

This case highlights the difficulties in diagnosing AIE and the value of measuring aciclovir and CMMG levels in making the diagnosis. ${ }^{4}$ In cases with renal failure and herpes simplex or varicella-zoster encephalitis, aciclovir should not be discontinued prematurely, but a total daily dose exceeding $400 \mathrm{mg}$ is not generally recommended in patients with renal impairment. Pharmacokinetic studies of aciclovir and CMMG are being undertaken at Karolinska University Hospital, Sweden, with the aim of achieving dose recommendations for patient with renal failure.

\section{Acknowledgements}

We are grateful to Dr M Snow, Department of Infectious Diseases, Newcastle General Hospital (NGH) for the clinical input and helpful comments, and Dr T J Walls (NGH) for financial support.

D Mahad, J Jarvis, P F Chinnery Department of Neurology, Newcastle General Hospital, Newcastle upon Tyne, UK

D Mitra, A Gholkar Department of Neuroradiology, Newcastle General Hospital

Division of Clinical Pharmacology, Karolinska Institute, Stockholm, Sweden

Correspondence to: Dr Don Mahad, Department of Neurology, Newcastle General Hospital, Newcastle upon Tyne, NE4 6BE; Don.Mahad@nuth.nhs.uk

doi: 10.1136/jnnp.2004.059824

Competing interests: none declared

\section{References}

1 Dorsky DI. Clyde S, Crumpacker S. Drugs five years later: acyclovir, Ann Intern Med 1987; 107:859-74.

2 Rashiq S, Briewa L, Mooney M, et al. Distinguishing acyclovir neurotoxicity from encephalomyelitis. J Intern Med 1993:234:507-11.

3 Braun JS, Apel I. Schaffer S, et al. Delirium during oral therapy of herpes zoster with acyclovir: case report and brief review of central nervous system side-effects of acyclovir. Nervenarzt 1998;69:1015-18.

4 Hellden A, Odar-Cederlof I, Diener P, et al. High serum concentrations of the acyclovir main metabolite 9-carboxymethoxymethylguanine in renal failure patients with acyclovir-related neuropsychiatric side effects: an observationa study. Nephrol Dial Transplant 2003; 18:1135-41.

5 Cohen SM, Minkove JA, Zebley JW, et al. Severe but reversible neurotoxicity from acyclovir. Ann Intern Med 1984;100:920

\section{Normal memory and no confabulation after extensive damage to the orbitofrontal cortex}

Subarachnoid haemorrhage caused by the rupture of an anterior communicating artery (ACoA) aneurysm is often followed by amnesia, confabulation, and personality change including social decision making..$^{1-3}$ However, the regions responsible for each symptom have not been determined conclusively. We describe a patient who showed personality change, but neither memory impairment nor confabulation, after extensive damage to the bilateral orbitofrontal cortex demonstrated by magnetic resonance imaging, providing evidence that the destruction of the medial orbitofrontal cortex alone cannot cause amnesia and confabulation.

\section{Case report}

The patient was a 45 year old, right handed man with a 16th grade education. He was not an apathetic person and worked hard as a manager before the onset. His past medical history was unremarkable and he had no medication. He had sudden onset of headache, became unconscious, and was admitted to an emergency hospital. Brain computed tomography showed a subarachnoid haemorrhage in the cisterns around the brainstem, longitudinal cerebral fissure, and bilateral Sylvian fissure caused by a ruptured aneurysm of the ACoA. On the same day, he underwent an operation to repair the ruptured aneurysm. There were slight brain oedema and vasospasm (four to 10 days). He did not become delirious, agitated, or suspicious. He had $200 \mathrm{mg}$ of phenitoin each day to prevent secondary seizures. His family noted that he showed mild anterograde amnesia, which improved over two months, but no retrograde amnesia.

The patient was discharged home after three months. He began working again as a manager at his company, but could not do his job as well as before the onset. Twenty months after the onset, he was admitted to our hospital for assessment of his problems.

On admission, the patient was fully alert and oriented. General physical and neurological examinations were unremarkable. During his stay in hospital, he had no problems communicating with others, kept his appointments and could find his way around the hospital. His family and his superior at his company reported that his personality had changed since the onset of his illness (in terms of lack of concern for others including his family, his appearance, and his future; the loss of spontaneity, initiative, and self motivation; disinhibition; and rigidity of thought).

General neuropsychological assessments were performed between the second and 12th hospital days. He was attentive, cooperative, and showed no confabulatory response. His intelligence level was normal on the Wechsler Adult Intelligence ScaleRevised (full IQ, 113; verbal IQ, 114; performance IQ, 109), Mini Mental State
Examination (30 of 30), and Raven Progressive Colored Matrices (35 of 36). He showed no linguistic deficit on the Western Aphasia Battery. The results of the Wisconsin Card Sorting Test (six categories achieved) and Verbal Fluency Test (animals, initial syllables "A", "Fu", and "Ni": 15, 10, 10, and 15/minute, respectively) were normal. His immediate memory spans were normal (forward: verbal, 7; spatial, 6; and backward: verbal, 6; spatial, 6). The indices on the Wechsler Memory Scale-Revised were above average, except for a somewhat low score for delayed index (general, 112; verbal, 110; visual, 108; attention/concentration, 112; delayed, 85). He showed no retrograde amnesia in a structured interview, on the Autobiographical Memory Interview (incidents 9, 9, 8 and personal semantic 21, 20, 21 for childhood, early adult life, and recent, respectively), and on the Public Events test (14, 15, 14, and 16/16 for 60th, 70th, 80th, and $90^{\text {th }}$, respectively).

Brain magnetic resonance imaging performed 20 months after the onset (fig 1) showed bilateral lesions in the ventromedial prefrontal lobe extending to the frontal pole and subcortical regions under the middle and superior frontal gyri. Additional lesions were seen in the left insula and the right ventral anterior nucleus of the thalamus.

\section{Discussion}

After extensive damage to the bilateral orbitofrontal cortex, with no concomitant lesion in the basal forebrain, the patient showed personality change as a result of a subarachnoid haemorrhage, but neither memory deficits on comprehensive neuropsychological assessment nor confabulation. His personality change could be classified as a combined type (apathetic and disinhibited) and was consistent with those (lack of concern, loss of spontaneity, disinhibition, impaired decision making, and rigidity of thought) generally agreed in the literature to be the result of dysfunction of the frontal lobe, particularly the orbitofrontal cortex.$^{1-3}$

Importantly, the patient showed no memory deficit. Damage to the basal forebrain without damage to the frontal lobe causes amnesia. ${ }^{4}$ With regard to the orbitofrontal cortex, it has been argued that destruction of this region is not necessary for the development of amnesia or basic cognitive function. ${ }^{13}$ However, there has so far been no conclusive evidence as to whether or not damage to the orbitofrontal cortex alone (especially the medial caudal part of it) gives rise to amnesia. ${ }^{1}$ Our present study provides evidence that damage to the orbitofrontal cortex alone does not result in amnesia and therefore strengthens the notion that the basal forebrain is one of the crucial sites for human memory.

It should be noted that the assessment of memory in our present study is based on standardised tests. This means that memory that is not measurable using these standardised tests (for example, temporal context memory) may be related to the function of the orbitofrontal cortex. In addition, we cannot draw a strong conclusion regarding frontal lobe function, because we did not use tests sensitive to damage to the ventromedial prefrontal cortex (for example, the Iowa Gambling Task ${ }^{3}$ ).

The patient showed no confabulation. Damage to the orbitofrontal lobe alone might not be sufficient for confabulation to be 

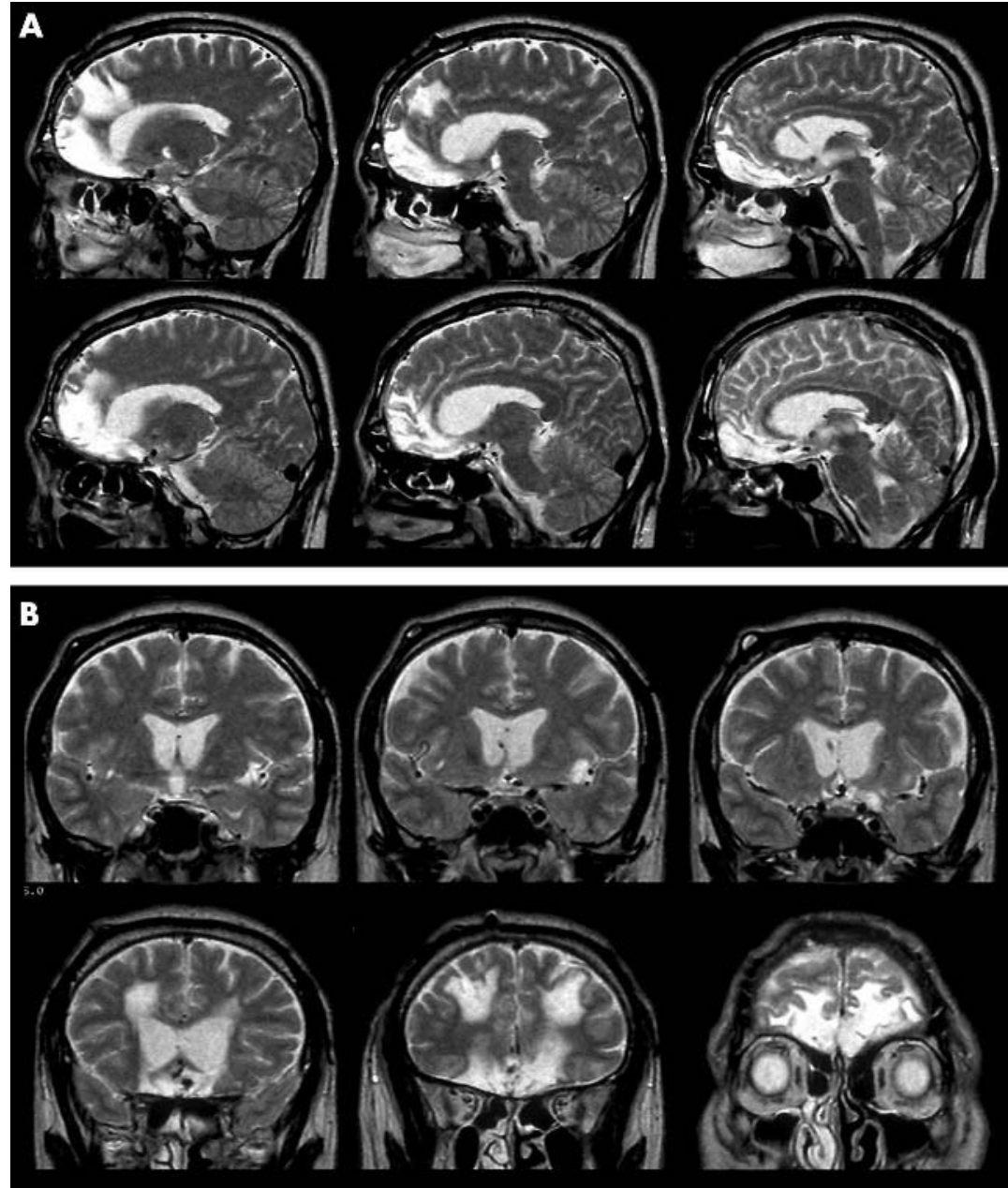

Figure 1 (A) Sagittal and (B) coronal T2 weighted magnetic resonance images showing extensive lesions in the bilateral orbitofrontal cortex. The core structures of the basal forebrain (medial septal nucleus, vertical and horizontal limb nuclei of Broca's diagonal band, and nucleus basalis of Meynert) were not damaged. In the sagittal sections, the images in the upper row show the right hemisphere and those in the lower row show the left hemisphere. In each row, the first, second, and third images are $15 \mathrm{~mm}, 10 \mathrm{~mm}$, and $5 \mathrm{~mm}$ away from the longitudinal cerebral fissure, respectively. In the coronal sections, the left side of the image corresponds to the right side of the brain. In the upper row, the first, second, and third images are $0 \mathrm{~mm}, 5 \mathrm{~mm}$, and $10 \mathrm{~mm}$ rostral from the anterior commissure. In the lower row, the first, second, and third images are $20 \mathrm{~mm}$, $30 \mathrm{~mm}$, and $50 \mathrm{~mm}$ rostral from the anterior commissure.

manifest. In one study, confabulation was seen in amnesic but not in non-amnesic patients with rupture of the ACoA and frontal lobe lesions, ${ }^{1}$ and in another study of amnesic patients with rupture of the ACoA, only those with frontal lobe lesions showed confabulation, ${ }^{5}$ suggesting that both frontal lobe lesions and amnesia are necessary for the development of confabulation. In contrast, a patient with confabulation and amnesia after damage to the basal forebrain but without frontal lobe damage has been reported. ${ }^{4}$ Further studies involving comprehensive neuropsychological and magnetic resonance imaging examination are needed to determine whether both frontal and basal forebrain involvement or basal forebrain involvement alone is required for the manifestation of confabulation.

T Fujii, M Suzuki, K Suzuki Department of Behavioural Neurology and Cognitive Neuroscience, Tohoku University Graduate School of Medicine, Sendai 980-8575, Japan; fujii@mail.tains.tohoku.ac.jp
H Ohtake

Department of Internal Medicine and Therapeutics, Yamagata University School of Medicine, Yamagato 990-2331, Japan

T Tsukiura

Neuroscience Research Institute, National Institute of Advanced Industrial Science and Technology, Tsukuba 305-8568, Japan

R Miura

Department of Psychiatry, Chubu National Hospital, Nagoya 474-8511, Japan

The patient gave informed consent for this letter to be published.

\section{doi: 10.1136/jnnp.2004.061846}

\section{References}

Competing interests: none declared

1 Deluca J, Diamond BJ. Aneurysm of the anterior communicating artery: a review of neuroanatomical and neuropsychological sequelae. J Clin Exp Neuropsychol 1995;17:100-12.
2 Arana FS, Parkinson JA, Hinton E, et al. Dissociable contributions of the human amygdala and orbitofrontal cortex to incentive motivation and goal selection. J Neurosci 2003;23:9632-8

3 Bechara A. The role of emotion in decisionmaking: evidence from neurological patients with orbitofrontal damage. Brain Cogn 2004:55:30-40.

4 Fukatsu R, Yamadori A, Fujii T. Impaired recall and preserved encoding in prominent amnesic syndrome: a case of basal forebrain amnesia. Neurology 1998:50:539-41.

5 Vilkki J. Amnesic syndromes after surgery of anterior communicating artery aneurysms. Cortex 1985;21:431-44.

\section{Adult onset SSPE: experiences in West Yorkshire over a 12 month period}

Subacute sclerosing panencephalitis (SSPE) is a rare delayed complication of measles virus infection in infancy. It is characterised by behavioural changes, myoclonus, cognitive impairment, visual disturbance, pyramidal and extrapyramidal signs, and ultimately coma leading to death. ${ }^{1}$ Typically, SSPE presents in childhood or early adolescence, but adult onset cases are recognised. Widespread measles immunisation in the UK has led to a dramatic fall in the incidence of SSPE in children, leading to the disease almost becoming extinct. ${ }^{2}$ However, a latent disease pool remains and cases may still come to the attention of adult neurologists, as borne out by our recent experience in West Yorkshire.

\section{Case histories}

An 18 year old man gave a three week history of blinking episodes lasting approximately one second, associated with a brief head jerk. These were not present in sleep. Examination revealed myoclonic jerks involving the neck associated with blinking. Initial electroencephalograms (EEGs), blood tests, and a magnetic resonance imaging scan were normal. Several anticonvulsant medications failed to suppress the jerks, which by four weeks had spread to the legs, causing unsteadiness. The mini mental test examination score at this stage was 26 of 30 . He began to deteriorate rapidly, with disorientation, blunted affect, dystonic posturing of the left arm, bradykinesia, and rigidity. Cerebrospinal fluid (CSF) was sent for analysis of 14-3-3 and S-100 proteins, which were negative. A further EEG, nine weeks after onset, demonstrated high voltage periodic complexes occurring every 10 seconds, consistent with SSPE. CSF and serum measles titres were raised at $35110 \mathrm{mIU} / \mathrm{ml}$ and $152930 \mathrm{mIU} / \mathrm{ml}$, respectively. The CSF to serum albumen ratio was $1: 300$, consistent with intrathecal antibody synthesis. There was no past history of measles, although he had received MMR (measles mumps rubella) immunisation at age 9. Oral inosiplex (isoprinosine) and subcutaneous interferon $\alpha 2 \mathrm{~b}$ were started and an Ommaya reservoir was inserted to administer intraventricular interferon $\alpha_{2} \mathrm{~b}$. By this stage, the myoclonus had subsided but he had gaze paresis, mutism, widespread spasticity, and required gastrostomy feeding. He received intraventricular treatment for six weeks before reservoir infection necessitated its removal. His condition plateaued and he was maintained on inosiplex alone. Eventually, he was discharged home in a dependent state. 
A 25 year old woman presented to her general practitioner complaining of impaired concentration, mood swings, disturbed sleep, and memory loss. One month later, she had noticed a fine tremor in both hands and occasional spasms affecting her right foot. Her concentration was worse and she mentioned word finding difficulties. She also had a tendency to stagger and fall. She was seen by a psychiatrist and somatisation was initially suspected. Later, she was referred to a neurologist. He noted that she had been acting oddly-for example, being found by her mother in a bath of cold water. Neurological examination was normal except for a mini mental test examination score of 20 of 30 with a child-like effect and stilted speech. A magnetic resonance imaging scan was unremarkable. She continued to deteriorate, developing right sided myoclonus. By this stage, she was unable to perform simple tasks, such as washing, and was aware of crawling sensations all over her body. Six months after her first presentation she had an EEG. This revealed repetitive complexes occurring every four to six seconds, often associated with a myoclonic jerk and consistent with SSPE. CSF analysis confirmed oligoclonal bands not present in the serum, which were positive for measles antibody antigen by immunoblotting. She had contracted measles at age 11 months. She was started on oral piracetam and inosiplex, along with subcutaneous interferon $\beta$ la, but she became bed bound with quadriparesis, dysarthria, and diffuse hyperalgesia. Her treatment was changed to intraventricular interferon

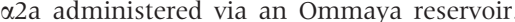
She required subcutaneous infusions of midazolam and diamorphine for symptom control, and nasogastric feeding was started. Her condition subsequently stabilised and she was discharged to a children's hospice.

\section{Discussion}

The estimated incidence of SSPE each year in developed nations is $<1 / 10$ million of the population under the age of 20 . The occurrence of two adult cases in the small region of West Yorkshire (population two million) within a few months of one another is remarkable. Although probably a chance finding, our experience emphasises the need for continued surveillance in populations where measles is no longer endemic. In theory more adult onset SSPE cases could present to neurologists in the future. This is because slow central nervous system spread of the virus over many years leaves open the potential for SSPE to present in later life, decades after population eradication of measles. In addition, routine immunisation has led to a shift in the incidence of measles towards unprotected children age $<1$, who are at a higher relative risk of developing SSPE in later life after a longer incubation period. ${ }^{2}$ Recently, there has been an increase in the number of measles outbreaks after a decline in the uptake of the combined MMR immunisation because of safety fears. ${ }^{3}$

In our patients, SSPE was not initially suspected, leading to diagnostic delay. Enquiring about measles in childhood may have been helpful in case 2 , but not case 1 , because infection in infancy was probably subclinical. In this patient the lack of typical findings on initial EEGs led to further delay, and instead we considered variant Creutzfeltd Jakob disease as a possibility. Patient 2 presented with early cognitive and behavioural changes. Initially, patients may come to the attention of other specialties with non-specific or psychiatric symptoms. ${ }^{4}$ It was only subsequent worsening of the patient's mental state associated with the development of unilateral myoclonic jerks that prompted further investigation, including EEG.

There is no curative treatment for SSPE. Trials have been complicated by variable natural history and spontaneous long remissions of the disease, although it is eventually fatal (median survival, three years). One study demonstrated similar response rates among patients randomised to inosiplex, with or without intraventricular interferon $\alpha 2 \mathrm{~b}$ treatment for six months (35\% v 34\%). ${ }^{5}$ However, these figures were substantially higher than historical remission rates of between $5 \%$ and $10 \%$. In our hands, intraventricular interferon was associated with initial worsening of encephalopathy and pronounced hyperpyrexia, possibly the result of chemically induced meningitis, and reservoir infection was a further complication. In both patients, disease appeared eventually to stabilise after antiviral treatment, particularly in patient 1 , in whom rapid progression at onset was suggestive of fulminant SSPE, usually fatal within three months.

Prevention seems to be the best approach, with mass immunisation leading to a drastic reduction in reported cases of measles and associated complications. However, our experience suggests that SSPE in adults should not be forgotten. Clinicians should remain vigilant for this devastating disease.

A Hassan, $O$ Lily, M Johnson Department of Neurology, St James's Hospital, Beckett Street, Leeds LS9 TTF, UK; ahamad@tiscali.co.uk

A Al-Din

Department of Neurology, Pinderfields Hospital, Aberford Road, Wakefield WFI 4DG, UK

doi: 10.1136/jnnp.2005.062828

Competing interests: none declared

\section{References}

1 Garg RK. Subacute sclerosing panencephalitis. Postgrad Med J 2002;78:63-70.

2 Miller C, Andrews N, Rush M, et al. The epidemiology of subacute sclerosing 2002. Arch Dis Child 2004;89:1145-8.

3 Jansen VA, Stollenwerk N, Jensen HJ, et al. Measles outbreaks in a population with declining vaccine uptake. Science 2003;301:804.

4 Salib EA. Subacute sclerosing panencephalitis (SSPE) presenting at the age of 21 as a schizophrenia-like state with bizarre dysmorphophobic features. Br J Psychiatry 1988;152:709-10.

5 Gascon GG. Randomized treatment study of inosiplex versus combined inosiplex and intraventricular interferon-alpha in subacute sclerosing panencephalitis (SSPE): international multicenter study. J Child Neurol 2003;18:819-27.

\section{Laterality of symptoms in patients} admitted to a stroke unit who had a discharge diagnosis of a psychiatric condition

Some psychiatric conditions produce symptoms that can mimic an acute neurological disease, including stroke. ${ }^{1-3}$ In several studies, such symptoms seemed to be more common on the left side of the body. ${ }^{1-3}$ The predominant processing of emotional information by the right hemisphere offers a hypothetical explanation for this finding.

We reviewed the discharge summaries of patients who were admitted to a stroke unit during the period May 1996 to December 2003 with a diagnosis of acute stroke and who had a discharge diagnosis of somatoform disorder and/or anxiety disorder according to the DSM-IV, revised ${ }^{4}$ criteria, and no recent stroke, according to the World Health Organisation definition. ${ }^{5}$

Two investigators (CS and LC) reviewed the discharge summaries independently and collected the following datain a standardised form: (a) age; (b) sex; (c) discharge psychiatric diagnosis (somatoform disorders: somatisation disorder and conversion disorder; anxiety disorders: generalised anxiety disorder and panic disorder); (d) type (motor paresis and/or involuntary movements, sensory, visual, other) and side (right, left, bilateral) of the symptoms; (e) vascular risk factors; $(f)$ neuroimaging data; and $(g)$ length of stay. Disagreements were solved by consensus.

\section{RESULTS}

From 2279 consecutively admitted patients to our stroke unit, we included 35 (1.5\%) discharge summaries for review. Of these, 25 patients had received a diagnosis of somatoform disorder (14 with somatisation disorder and 11 with conversion disorder), and 7 patients had a diagnosis of anxiety disorder (4 with generalised anxiety disorder and 3 with panic disorder). Three patients had other psychiatric diagnoses. Symptoms were presented on the left side of the body in ll patients, on the right side of the body in 14, and 10 presented bilateral symptoms (table 1$)$. There were 21 patients $(60 \%)$ with vascular risk factors, of whom 11 (31.4\%) had more than one risk factor. Median hospitalisation stay was 3 days.

There were no statistical differences in demographic variables, discharge psychiatric diagnosis, type of the symptoms, vascular risk factors, neuroimaging data, or length of stay between patients with left sided symptoms and those with right sided symptoms concerning (table 1). Patients with somatoform disorders had motor symptoms more frequently than did patients with anxiety disorders $\left(\chi^{2}=6.84 ; \mathrm{p}=0.02\right)$.

\section{DISCUSSION}

In this study, $1.5 \%$ of the patients admitted to a stroke unit presented symptoms unexplained by stroke or other neurological disease and fulfilling the criteria for psychiatric diagnosis. Contrary to most of published studies, our results did not show a preponderance of left sided symptoms. In a metaanalysis of all studies describing patients with medically unexplained symptoms, Stone et al concluded that is not certain that the functional symptoms are more common on the left side than on the right side of the body. They found a preponderance of the left symptoms only in the studies in which the investigators were aware of the laterality hypothesis before performing the study. ${ }^{1}$ As in that meta-analysis, we did not find a preponderance of left sided symptoms.

The younger median age and the female preponderance of patients with psychiatric conditions mimicking a stroke reflects the demographic characteristics of somatoform 
Table 1 Differences between patients with a psychiatric diagnosis and with left and right sided symptoms

\begin{tabular}{|c|c|c|c|c|}
\hline Variables & n (\%) & Left group & $\begin{array}{l}\text { Right } \\
\text { group }\end{array}$ & $p$ value \\
\hline \multicolumn{5}{|l|}{ Demographics } \\
\hline Age (median) & 41.0 & 41.0 & 38.0 & $0.94^{*}$ \\
\hline $\operatorname{Sex}(F / M)$ & $\begin{array}{c}28(80.0) / \\
7(20.0)\end{array}$ & $\begin{array}{l}9(81.8) / \\
2(18.2)\end{array}$ & $\begin{array}{c}10(71.4) / \\
4(28.6)\end{array}$ & $0.66+$ \\
\hline \multicolumn{5}{|l|}{ Diagnosis } \\
\hline Somatoform disorders & $25(71.4)$ & $9(81.8)$ & $10(90.9)$ & - \\
\hline Somatisation disorder & $14(40.0)$ & $4(36.4)$ & $7(63.6)$ & $1.00+$ \\
\hline Conversion disorder & 11 (31.4) & $5(45.5)$ & $3(27.3)$ & - \\
\hline Anxiety disorders & $7(20.0)$ & $2(18.2)$ & $1(9.1)$ & - \\
\hline GAD & $4(11.4)$ & $1(9.1)$ & $1(9.1)$ & - \\
\hline Panic disorder & $3(8.6)$ & $1(9.1)$ & 0 & - \\
\hline Other & $3(8.6)$ & - & 3 & - \\
\hline \multicolumn{5}{|l|}{ Symptoms } \\
\hline \multicolumn{5}{|l|}{ Paresis } \\
\hline Total & $26(74.3)$ & $9(81.9)$ & $12(85.7)$ & $1.00 \dagger$ \\
\hline Left & $10(38.5)$ & - & - & - \\
\hline Right & $12(46.2)$ & - & - & - \\
\hline Bilateral & $4(15.4)$ & - & - & - \\
\hline \multicolumn{5}{|l|}{ Involuntary movements } \\
\hline Total & $1(2.9)$ & 0 & $1(7.1)$ & $1.00 \dagger$ \\
\hline Right & 1 & - & - & - \\
\hline \multicolumn{5}{|l|}{ Sensory } \\
\hline Total & $17(48.6)$ & $4(36.4)$ & $7(50.0)$ & $0.69+$ \\
\hline Left & $4(23.5)$ & - & - & - \\
\hline Right & $7(41.2)$ & - & - & - \\
\hline Bilateral & $6(35.3)$ & - & - & - \\
\hline \multicolumn{5}{|l|}{ Visual } \\
\hline Total & 5 (14.3) & $1(9.1)$ & $2(14.3)$ & $1.00 \dagger$ \\
\hline Bilateral & 5 & - & - & - \\
\hline \multicolumn{5}{|l|}{ Other } \\
\hline Total & 19 (54.3) & $3(27.3)$ & $9(64.3)$ & $0.15+$ \\
\hline \multicolumn{5}{|l|}{ Total } \\
\hline Left & $11(31.4)$ & 11 & - & - \\
\hline Right & $14(40.0)$ & - & 14 & - \\
\hline Bilateral & $10(28.6)$ & - & - & - \\
\hline
\end{tabular}

and anxiety disorders. One interesting finding was the high frequency of vascular risk factors, which increases diagnostic uncertainty and could explain the admission to the stroke unit. A major limitation of our study is the lack of use diffusion magnetic resonance imaging to exclude definitively the unlikely possibility of a concomitant ischaemic lesion.

We conclude that left sided laterality of symptoms cannot be used as a tool to establish a psychiatric diagnosis in patients with acute lateralised neurological symptoms.

C O Santos, L Caeiro, J M Ferro

Stroke Unit, Serviço de Neurologia, Department of Neurosciences and Mental Health, Hospital de Santo Maria, Faculdade de Medicina de Lisboa, Portugal

R Albuquerque, M L Figueira Psiquiatria, Department of Neurosciences and Mental Health, Hospital de Santa Maria, Faculdade de Medicina de Lisboa, Portugal

Correspondence to: $\operatorname{Dr} C \mathrm{O}$ Santos, Serviço de Neurologia, Hospital de Santa Maria, 1649-035 Lisboa, Portugal; acosta@fm.ul.pt

doi: $10.1136 /$ jnnp. 2005.062893

Competing interests: none declared

\section{REFERENCES}

1 Stone J, Sharpe M, Carson A, et al. Are functiona motor and sensory symptoms really more frequent on the left? A systematic review. J Neurol Neurosurg Psyquiatry 2002;73:578-81. motor symptoms: frequency of neurological pathology depends on side of symptoms. J Neurol Neurosurg Psychiatry 1994;57:1401-2.

3 Ron MA. Somatisation in neurological practice. J Neurol Neurosurg Psychiatry 1994;57:1161-4.

4 American Psychiatric Association. Mood disorders. Diagnostic and statistical manual of mental disorders, 4th ed. Washington DC: American Psychiatric Press, 2002.

5 WHO MONICA Project Principal Investigators. The World Health Organization MONICA Project (monitoring trends and determinants in cardiovascular disease): a major international collaboration. WHO MONICA Project Principal Investigators. J Clin Epidemiol 1988;41:105-14.

\section{BOOK REVIEW}

\section{Atlas of neuromuscular diseases}

Edited by Eva L Feldmand, Wolfgang Grisold, James W Russell, Udo Zifko. Published by Springer Verlag, 2005, €198 (hardcover), pp 474. ISBN 3-211-83819-8

This book it titled an "atlas" and subtitled "a practical guideline". I had the expectation of a heavily illustrated book of neuromuscular conditions with annotations. The book has been designed as a comprehensive coverage
2 Rothwell P. Investigation of unilateral sensory or of disorders of peripheral nerve (including cranial nerves), muscle, neuromuscular junction, and motor neurone. Each condition is outlined in note form, with particular emphasis on causation - which can be lengthy and repetitive. The style of the text is abbreviated and can be difficult to read. The précis can be misleading or inaccurate at times. A useful list of references is given.

It is not clear to me to whom this book would appeal. The text is probably too comprehensive and potentially misleading or confusing for students, and does not add much to those already informed. The number and quantity of the illustrations is disappointing. I had expected more clinical illustrations of the conditions described. As a practical guideline it is not helpful and in particular the therapeutic suggestions are too abbreviated, incomplete, and uncritical for clinical application. Problems to be addressed in the next edition include the use of the same nerve biopsy illustration for patients with chronic inflammatory demyelinating polyneuropathy and Charcot-Marie-Tooth disease, despite one image being rotated by 90 degrees; the illustration of text relating to genetically defined young onset spinal muscular atrophy with an elderly man who appears to have progressive muscular atrophy; and the spelling of fascioscapulohumeral (sic) dystrophy.

The authors point out that "no other book provides a complete overview in a structured and easily comprehensive pattern supported by figures and pictures". There are, however, a number of excellent books related to diseases of muscle and nerve, some providing a wider range of clinical and pathological illustrations and a more critical review of clinical presentation, diagnosis, and treatment. At the present time the role claimed by this book remains unfilled.

R W Orrell

\section{CORRECTION}

doi: 10.1136/jnnp.2002.003392

M S Welgampola, S M Rosengren, G M Halmagyi, et al. Vestibular activation by bone conducted sound ( $J$ Neurol Neurosurg Psychiatry 2003;74:771-8). This relates to calibration of the output of the bone conductor that was used. The audiometric calibrator was not fully aware of the specific units in which bone conductors are generally calibrated but rather was influenced by the units of the sound level meter (suitable for air conducted sound). The calibration procedure is normally performed purely for testing hearing, in which case the precise units used are not strictly relevant (and under conditions which are quite different from those that the authors used). The authors have now purchased the required equipment to allow calibration of the B7l, and have provided a guide to the force levels obtained:

Bone conducted stimuli should be measured in units of dB FL (force level) as a ratio to a reference force of $1 \mu \mathrm{N}$. These levels are 10-20 dB higher, depending upon frequency, than the values given as "SPL". The standard parameter $500 \mathrm{~Hz} 7 \mathrm{~ms}$ " $112 \mathrm{~dB}$ SPL" tone burst stimulus, obtained with a $10 \mathrm{~V}$ peak to peak input, corresponds to an intensity of 127 dB FL (RMS). 